\title{
GNSS Signals Acquisition and Tracking in Unfavorable Environment
}

\author{
Saifeddine CHEBIR, Salih AIDEL, Khaled ROUABAH, Salim ATIA, Mustapha FLISSI
}

ETA Laboratory, Electronics Dept., University of Mohamed El Bachir El-Ibrahimi, Bordj Bou Arreridj- Algeria

khaled_rouabah@yahoo.fr

Submitted August 16, 2017 / Accepted March 10, 2018

\begin{abstract}
In this paper, we propose a method based on applying specific transformations to the Global Navigation Satellite System (GNSS) signals received in unfavorable environment. As a result, one simple classical receiver including these adjustments becomes sensitive to several Multi-Constellation and Multi-Frequency (MC/MF) GNSS signals and achieves efficiently their collective acquisition. The proposed method consists of three variants each dedicated to a particular type of Binary Offset Carrier (BOC) family signals; the primary is based on undersampling process, the second is founded on time expansion and the last one permits the acquisition of more than five different GNSS signals by a single local Composite Binary Coded Symbols (CBCS) waveform replica. Hence, the proposed scheme, by avoiding the use of multiple demodulators in the baseband, allows less receiver complexity and accordingly better realization cost. The simulation results showed that the proposed method presents an effective solution for the reception of $M C / M F$ signals in unfavorable environments.
\end{abstract}

\section{Keywords}

GNSS, Galileo, correlation, multi-constellation, multi-frequency, multipath

\section{Introduction}

Currently, the GNSS positioning is widely used in many areas such as vehicles, aviation, navy and other various applications. The transmission and multiplexing method adopted by the most of these systems is the Code Division Multiple Access (CDMA) based on the Direct Sequence Spread Spectrum (DSSS) technique [1]. The Binary Phase Shift Keying (BPSK) modulation was firstly used for traditional GNSS [1]. Afterwards, for reasons of improved accuracy and cohabitation between traditional and new generation GNSS, a new modulation called BOC was introduced and approved [2]. The latter is more effective since it presents better performances with specific constraints such as multipath (MP), noise, low power, interferences, interoperability and compatibility [2].
Further developments on the BOC modulation conducted on several other types of modulations, which constitute nowadays the BOC modulation family. Namely, we can cite the Multiplexed BOC (MBOC) modulation developed for L1C modernized Global Positioning System (GPS) and Galileo Open Service (OS) signals [3]. It has two implementations: the first one is a four-level signal called Composite BOC (CBOC) [4] and the second one is a bi-level signal called Time-Multiplexed BOC (TMBOC) [3]. One more modulation called Binary Coded Symbol (BCS), which is a generalization of BPSK and BOC modulations, has been presented in [5]. The BCS waveform has a sharp Auto-Correlation Function (ACF) that results in very good performance in terms of natural MP rejection and high flexibility for future GNSS optimization [6]. BCS was followed by the Composite Binary Coded Symbols (CBCS) that is developed in [5], [7] as a candidate for L1 OS optimized signal structure. Its concept is similar to that of MBOC signal and can be expressed by a linear combination of $\operatorname{BOC}(\alpha, \alpha)$ and a BCS waveform with the same chip rate [7]. Based on BCS principle, other BOC family members are the BOC with Adjustable Width (BOC-AW) modulation and its Optimized version (OBOC-AW) that are characterized, respectively, by three levels $(-1,0,1)$ and two levels [8]. BOC-AW and OBOC-AW waveforms present better resistance to noise, jamming and interferences, compared to the traditional BOC and BCS ones for the same receiving band [8]. Newly, consistent BCS modulation sequences are proposed in [9] providing high performance in terms of code tracking efficiency, and interference and MP mitigation.

As can be seen from the preceding parts, GNSS receivers' performances are hampered by some problems such as MP [1], [10], interferences and noise effects [1]. The MP signals are characterized by their number, amplitudes, delays and phases, and constitute a source of overriding error [11]. In effect, the MP creates a bias in the estimation of the time delay by a GNSS receiver's Delay Locked Loop (DLL), which results in a positioning error [12]. Several studies- have been carried out to accomplish a thorough investigation on the principal causes of GNSS performances degradation and provide adequate solutions to their effects. Several techniques were suggested in the literature for MP reduction. For example, in [13], a descrip- 
tion of traditional MP mitigation methods is presented, developed for existing GNSS signals, namely, the narrow correlator [14], the Double Delta Correlator [13], the Enhanced Double Delta Correlator [15], the Early-Late Slope technique [13] and the Early1/Early2 Tracker [13]. In addition, several other complex but powerful techniques reducing the effect of MPs are found in literature in the case of complete absence of side peaks in the ACF. Among these, we can cite as examples: the Multipath Estimation Techniques [16], the Multipath Estimating DLL (MEDLL) [17], the Teager-Kaiser-Operator-Based MEDLL [18], the Fast Iterative Maximum Likelihood Algorithm (FIMLA) [19] and the Virtual MP Mitigation (VMM) [20].

In GNSS systems, MPs do not represent the unique problem. In fact, when using BOC modulation, another drawback appears because of the presence of multiple side peaks in the shape of the ACFs of BOC modulated signals. These side peaks may create false locks at the receiver DLL loop causing ambiguity in the tracking process [21]. Consequently, several side peaks cancellation techniques were also proposed in the literature. As examples, we can cite Autocorrelation Side-Peak Cancellation Technique (ASPeCT) [22], Sidelobes Cancellation Method (SCM) [23], and General Removing Ambiguity via Side Peak Suppression (GRASS) method for only BOCsin signals [24]. We can mention also Simultaneous Perturbation Stochastic Approximation (SPSA) for BOCcos signals [25], No Central Peak ACF for BOCsin signals [26], Pseudo Correlation Function method for MBOC signals [24], Combined Correlation Functions method for BOCsin/cos, CBOC and Alternate BOC signals [27] and BOC-PRN (Pseudo Random Noise) method for BOCsin/cos signals [28].

In addition to the aforementioned limitations, the reception of weak GNSS signals is another challenging problem that is becoming ever more common due to the massive use of satellite localization devices [29], [30]. The GNSS signal in indoor locations and weak signal environments, like deep urban canyons is nominally $10-25 \mathrm{~dB}$ weaker than the lower bound of $-130 \mathrm{dBm}$ observed in open-sky conditions [31]. To enhance the acquisition process sensitivity in such conditions, the long coherent or incoherent integration is usually an efficient method [30]. The coherent integration decreases the noise bandwidth and improves sensitivity, but it is limited by the unknown data bits and bit edges [30]. Meanwhile, the incoherent integration, even though it does not suffer from such limitations, is less sensitive because of squaring loss, and it becomes less effective as its number increases [30].

Furthermore, the implementation of GPS receiver algorithms for weak signals is confronted to the limited resources of the wireless devices. Hence, any practical algorithm should be designed in accordance to the requirements of such devices. For this purpose, several algorithms have been developed in [32], [33]. The new generation GNSS are using signals with equal codes and bits periods which introduces another severe limitation caused by the presence of bit transition in each segment of the received signal. The bit transition, provoked by the navigation data or secondary code, may cause the splitting of the main peak of the Cross-Ambiguity Function (CAF) into two smaller lobes along the Doppler shift axis. This problem, in weak signal condition, results in a biased estimation of the code delay and the Doppler shift [34]. Among the first methods proposed to overcome the bit transition problem is that based on the energy invariance property of the CAF [35]. The authors in [36] proposed a novel two-step based bitsign transition cancellation method. The latter, even though it permits improved performance over conventional acquisition approach, requires a greater computational load to perform the acquisition process. Another interesting method called Double Bloc Zero Padding (DBZP) has been presented in [37]. This is shown to be sensitive to bit transition and computationally efficient while conserving the same Signal to Noise Ratio (SNR) at the DBZP output as that at the output of the traditional correlator. The DBZP method was then followed by the Modified Time Parallel Acquisition scheme based on the Fast Fourier Transform (FFT) algorithm and proposed in [38] to solve the transition problem. Here, both coherent and non-coherent accumulation methods have been tested after bearing some changes [39]. It has been shown that the coherent processing outperforms its non-coherent counterpart at the expense of an increased computation load. Afterwards, in [40], in terms of detection probability, a thorough study on the bit-sign transition impact on the acquisition performance was conducted. This work ended up determining the optimal acquisition parameters in presence of bit-sign transitions. In [41], the same authors, by taking into account the bit sign transition, provided a general mathematical study of the acquisition, expressed the probability of detection and evaluated the average detection probability.

In addition to the bit transition drawback, the satellitemasking problem, especially in urban environment, represents a serious problem. Indeed, in this case, users can find themselves in places where some satellites beyond the horizon are actually masked by obstacles. This loss of visibility of certain satellites degrades the Dilution Of Precision (DOP) available for the user and the accuracy of the service [10], [42]. To overcome this disadvantage, the researchers made use of the benefits provided by the coexistence of several different GNSS such as GPS, Globalnaya Navigatsionnaya Sputnikovaya Sistema (GLONASS) or "Global Navigation Satellite System" and the new Galileo and Compass systems, when designing a MC/MF receiver. Accordingly, many research efforts have been aimed at the development, implementation and design of MC/MFGNSS software and hardware defined receivers. Nevertheless, it should be noted that MC systems introduce an additional unknown to the estimation procedure due to the offset between their time scales [43]. Accordingly, the required number of visible satellites increases [43]. To resolve this problem, an algorithm has been proposed in [44] to obtain a position solution with only four visible GPS/GLONASS satellites. However, the experimental assessment results of this algorithm showed that a position solution could be obtained at a slight loss in accuracy. In 
addition, in [45] the combined GPS-Galileo navigation solution has been assessed providing slight improvements with respect to the standalone GPS. In addition, the results presented in [43] confirm that the GPS/GLONASS combination, reduced to only four combined satellites, shows apparent improvements compared to GPS-only solution in terms of availability and accuracy.

In this paper, we propose a method for receiving GNSS signals in an unfavorable environment. The latter one presents less computational load and is characterized by its ability to acquire and track several MC/MF GNSS signals using a simple classical receiving structure. The simulation results, based on Matlab, showed that the proposed method presents a better performance against MPs and noise in urban environments and thus it can be considered as a likely solution for the reception of $\mathrm{MC} / \mathrm{MF}$ signals.

The rest of the paper is organized as follows. In Sec. 2, brief descriptions of different categories of signals that can be received by our structure are given. Section 3 describes the proposed reception architecture in detail. Section 4 presents and discusses the simulation results of the proposed method. Finally, conclusions are presented in Sec. 5.

\section{GNSS Modulations}

The baseband modulated signal $y(t)$ used by a noiseless GNSS can be given as the product of the navigation message $d(t)$, the spreading code $c(t)$ (PRN code with chip duration $\left.T_{\mathrm{c}}\right)$, and the subcarrier signal $s(t)$ :

$$
y(t)=d(t) c(t) s(t) .
$$

In the GPS C/A signal, $s(t)$ equals one and $y(t)$ is BPSK modulated with a chipping rate $f_{\mathrm{c}}=1 / T_{\mathrm{c}}=f_{0}=1.023 \mathrm{MHz}$ [1].

The $\operatorname{BOC}(\alpha, \beta)$ modulation originally proposed by Betz in [2] and adopted by Galileo and GPS modernization uses a square waveform subcarrier with different values of subcarrier frequency $f_{\mathrm{s}}=\alpha f_{0}$ and $\mathrm{PRN}$ code rate $f_{\mathrm{c}}=\beta f_{0}$ where $\alpha$ and $\beta$ are positive integers.

There are two versions of BOC modulation, BOCsin and $\mathrm{BOCcos}$, which are related respectively to the sine and cosine phased subcarrier square waveforms that are typically defined by [2], [46]:

and

$$
S_{\sin }(t)=\operatorname{sign}\left[\sin \left(2 \pi f_{\mathrm{s}} t\right)\right]
$$

$$
S_{\mathrm{cos}}(t)=\operatorname{sign}\left[\cos \left(2 \pi f_{\mathrm{s}} t\right)\right] \text {. }
$$

The $\operatorname{MBOC}(\alpha, \beta, p)$ modulation is the optimized version of BOC modulation proposed for Galileo L1-OS signal and the modernized GPS L1C signal [3]. The designation $\operatorname{MBOC}(\alpha, \beta, p)$ indicates that the Power Spectral Density (PSD) is a combination of $\operatorname{BOC} \sin (\alpha, \beta)$ and
$\operatorname{BOC} \sin (\beta, \beta)$ subcarriers with different power distributions $p$ and $(1-p)$, respectively, such as [3]:

$$
\mathrm{G}_{\mathrm{MBOC}}(f)=(1-p) \mathrm{G}_{\mathrm{BOC}(1,1)}(f)+p \mathrm{G}_{\mathrm{BOC}(6,1)}(f) \text {. }
$$

There are two possible implementations of the MBOC modulation, the CBOC for Galileo L1-OS with split power $(50 \% / 50 \%)$ between the data component and the pilot component and the TMBOC GPS L1C signal with $75 \%$ power on the pilot component and $25 \%$ power on the data component [3], [4]:

The subcarrier signal $s(t)$ in the CBOC implementation is the weighted sum of BOCsin $(1,1)$ and BOCsin $(6,1)$ subcarriers spreading symbols given by [3], [4]:

$$
\mathrm{s}(t)=\sqrt{1-p} \operatorname{sign}\left[\sin \left(2 \pi f_{0} t\right)\right]+\sqrt{p} \operatorname{sign}\left[\sin \left(12 \pi f_{0} t\right)\right]
$$

where $p=1 / 10$.

In the $\operatorname{TMBOC}(6,1,4 / 33)$ implementation, the data component subcarrier signal is the BOCsin $(1,1)$ spreading symbols, while the pilot component one, denoted $s_{\text {pilot }}(t)$, comprises 29/33 BOCsin(1,1) and 4/33 BOCsin(6,1) spreading symbols, such that :

$$
S_{\text {pilot }}(t)= \begin{cases}\operatorname{sign}\left[\sin \left(2 \pi f_{0} t\right)\right] & \text { if } t \in S_{1} \\ \operatorname{sign}\left[\sin \left(12 \pi f_{0} t\right)\right] & \text { if } t \in S_{2}\end{cases}
$$

where $S_{1}$ and $S_{2}$ are the set of chips where the subcarrier is of the $\mathrm{BOC} \sin (m, n)$ and $\mathrm{BOC} \sin (n, n)$ sort, respectively.

The subcarriers signals for BPSK, BOC or MBOC can be generated from the spreading symbols of BCS signals, denoted by $s_{T_{\mathrm{C}}}(t)$, and given by the following equation $[5],[6],[7]$ :

$$
s_{T_{\mathrm{C}}}(t)=\sum_{k=0}^{n-1} S_{k} P_{T_{\mathrm{C}} / n}\left(t-k \frac{T_{\mathrm{C}}}{n}\right)
$$

where $P_{T_{\mathrm{c}} / n}$ represents the rectangular pulse function of duration $T_{\mathrm{C}} / n$ that is defined by:

$$
P_{\Delta}(t)=\left\{\begin{array}{lrr}
1 & \text { for } & 0 \leq t \leq \Delta \\
0 & & \text { otherwhise }
\end{array}\right.
$$

and $S_{k}$ is the sequence with values +1 or -1 .

For GNSS BCS signals, the notation $\operatorname{BCS}\left([\mathrm{s}], f_{\mathrm{c}}\right)$ is used, where [s] represents the BCS sequence in one chip and $f_{c}$ is the chip rate [5], [7].

According to [5], [7], BPSK and BOC signals are particular cases of the $\operatorname{BCS}$ modulation. Thus, the $\operatorname{BPSK}(\beta)$ modulation is equivalent to a $\operatorname{BCS}\left([1,1, \ldots, 1], f_{\mathrm{c}}\right)$, where the values of $\beta$ can be chosen arbitrarily. In a similar way, $\operatorname{BOC}(\alpha, \beta)$ modulation is equivalent to a $\operatorname{BCS}([1,-1,1,-1$, $\left.\ldots, 1,-1], f_{\mathrm{c}}\right)[5],[6],[7]$. And the $\operatorname{MBOC}(6,1,1 / 11)$ subcarrier signal is a particular case of the optimized version of the BCS, denoted CBCS when the BCS sequence [s] is selected with the BOCsin signal [5]. 
The CBCS subcarrier signal is defined by a superposition of the BOC and BCS subcarrier signals given by [5], [7], [47]:

$$
s(t)=p_{1} \cdot \operatorname{sign}\left[\sin \left(2 \pi \alpha f_{0} t\right)\right]+q_{1} \cdot s_{T_{\mathrm{C}}}(t)
$$

where $p_{1}$ and $q_{1}$ are the coefficients that define power distributed over each component and satisfying the relation: $p_{1}^{2}+q_{1}^{2}=1$. In general, the CBCS modulation is denoted by $\operatorname{CBCS}([1,-1,1,-1,1,-1,1,-1,1,1], 1,20 \%)$ or $\operatorname{CBCS}(20 \%)$. where, $\{[1,-1,1,-1,1,-1,1,-1,1,1]\}$ is a vector corresponding to the BCS sequence. The value " 1 " before $20 \%$ corresponds to the case where $f_{\mathrm{c}} / f_{\mathrm{S}}=1$. " $\%$ " represents the percentage BCS power relative to the total power signal. The amplitude of the BCS component $q_{1}$ is weaker than that of the BOCsin $(1,1)$ to remain closer to the BOCsin(1,1) base line signal [5], [7].

\subsection{Autocorrelation Functions}

The receiver signal processing performance is strongly related to the shape of the ACF between the received and the locally generated signals. This ACF is computed between the incoming $S(t)$ modulated signal and its corresponding locally generated replica $S_{1}(t)$. It can be given as follows:

$$
R(\tau)=\int_{-\infty}^{+\infty} S(t) S_{1}(t-\tau) \mathrm{d} t
$$

In GNSS applications, there are two categories of BOC modulated signals; the first one characterizes signals of low-order BOC modulation while the second one represents those of high-order BOC modulation.

In Fig. 1, the normalized ACF of BPSK(1) modulated signal is plotted together with those of the first category of BOC modulated signals: BOCsin $(1,1), \operatorname{CBOC}(6,1,1 / 11,+)$, TMBOC $(6,1,4 / 33), \quad \mathrm{BOC} \cos (1,1)$ and $\mathrm{CBCS}(20 \%)$. As shown in this figure, the $\operatorname{BPSK}(1)$ ACF presents one

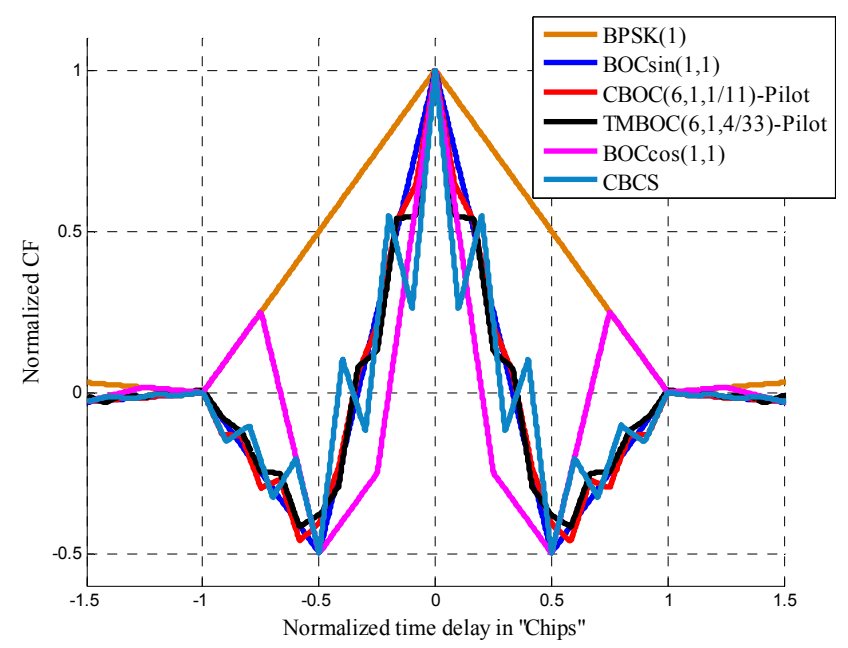

Fig. 1. Normalized ACFs of $\operatorname{BPSK}(1), \operatorname{BOCsin}(1,1)$, $\operatorname{CBOC}(6,1,1 / 11,+), \operatorname{TMBOC}(6,1,4 / 33), \operatorname{BOC} \cos (1,1)$ and $\operatorname{CBCS}(20 \%)$.

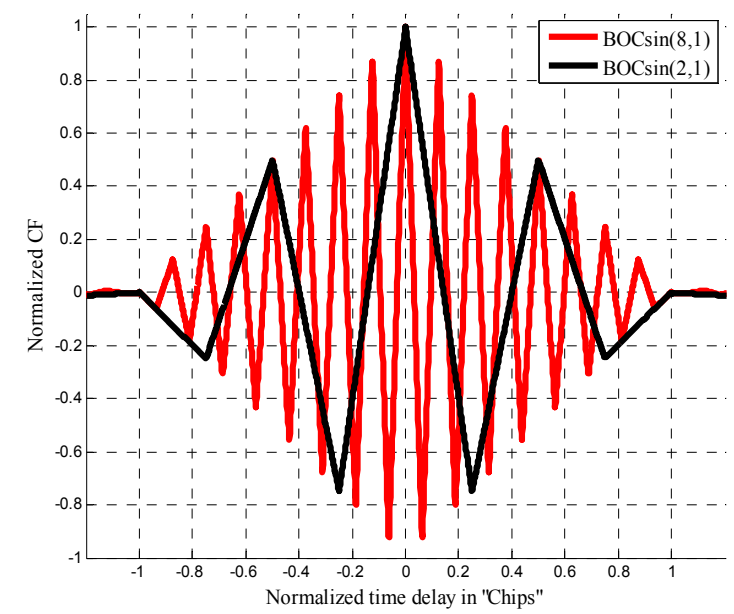

Fig. 2. Normalized ACFs of BOCsin $(2,1)$ and BOCsin $(8,1)$.

central peak of large width and a total absence of side peaks, while each of the remaining ACFs presents a central main peak and several side ones. $\operatorname{CBOC}(6,1,1 / 11,+)$, TMBOC $(6,1,4 / 33)$ and $\mathrm{CBCS}(20 \%)$ ACFs all present a sharper principal peak and have side peaks with smaller levels compared to those of $\mathrm{BOC}(1,1)$. These advantages are due to the amount of power translated towards higher frequencies [3], [5], [48].

In Fig. 2 that characterizes the second category, we present ACFs for two different high-order BOC modulated signals. As illustrated in this figure, each normalized ACF has a central main peak and several side ones with considerable levels. As illustrated in Fig. 2, the number of side peaks in $\operatorname{BOC}(\alpha, \beta)$ modulated signals increases with the modulation order $N=2 \alpha / \beta$ [2].

\subsection{Power Spectral Density}

Figure 3 depicts the PSDs of BPSK(1), BOCsin(1,1), $\operatorname{CBOC}(6,1,1 / 11,+), \operatorname{TMBOC}(6,1,4 / 33), \operatorname{BOC} \cos (1,1)$, and CBCS $(20 \%)$ modulated signals. As shown in this figure, the PSDs of BOCsin/ $\cos (1,1)$ modulated signals has each two symmetrical main lobes centered at the frequencies

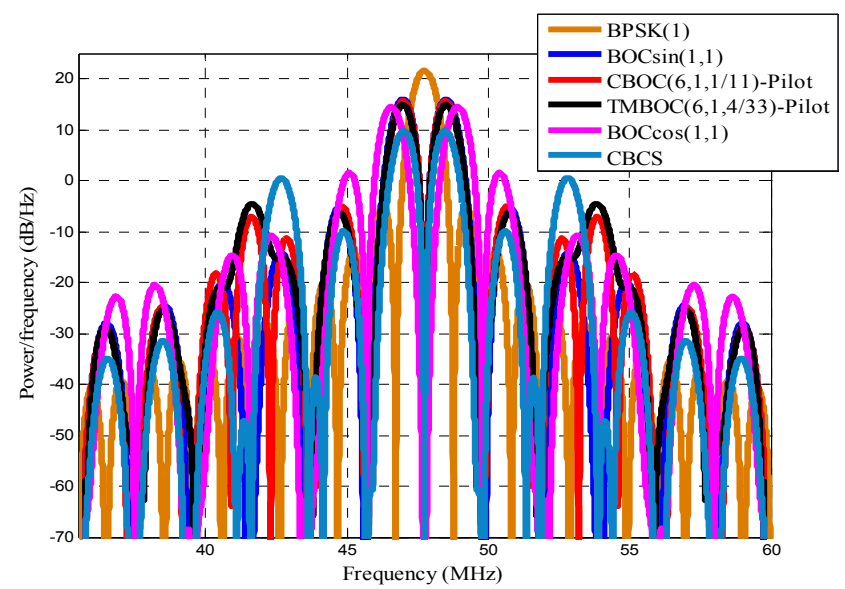

Fig. 3. Normalized PSDs of $\operatorname{BPSK}(1), \operatorname{BOCsin}(1,1)$, CBOC $(6,1,1 / 11,+), \quad \operatorname{TMBOC}(6,1,4 / 33), \operatorname{BOC} \cos (1,1)$ and $\operatorname{CBCS}(20 \%)$. 


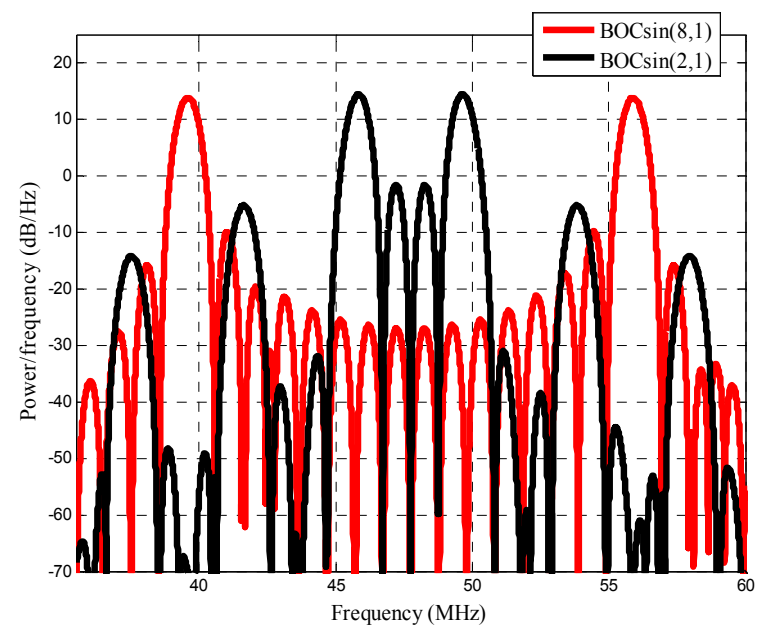

Fig. 4. Normalized PSDs of BOCsin $(8,1)$ and BOCsin $(2,1)$.

$f_{\mathrm{IF}}=47.74 \mathrm{MHz}+f_{0}$. In addition, and in comparison to this latter case, as shown in the same figure, the CBOC, TMBOC and CBCS signals' PSDs have two symmetrical main lobes with increased power for their side lobes and additional zeros. These characteristics confirm their resistance to noise and MP.

Figure 4 describes the PSDs of the second category BOCsin $(2,1)$ and BOCsin $(8,1)$ modulated signals. As illustrated in this figure, both of these PSDs have symmetrically spaced main lobes. The spacing between these latter is proportional to the modulation order.

\section{Principle of the Proposed Method}

Positioning is seriously affected by the presence of weak power signals at the GNSS receiver's antenna. Besides, the use of such signals in classical reception methods is unavoidable, since the number of available signals, which are then confined to a single constellation, is limited. The design of MC/MF-GNSS software and hardware defined receivers brings considerable advantages to Position Velocity and Time (PVT) calculator that can be summarized as follows [49], [50]:

- Improved position and time accuracy with increased number of satellites compared to single system use;

- Highly strengthened resistance to masking (in urban areas);

- Enhanced DOP due to the improved spatial distribution of visible satellites;

- Suppression of the ionospheric error in the position calculation by comparing the delays of two GNSS signals;

- Improvement of robustness against interferences by using different frequency bands.

The proposed method uses a receiver acquisition and tracking process that benefits from all the sub mentioned advantages; it consists of three variants used according to the type of the received signals.

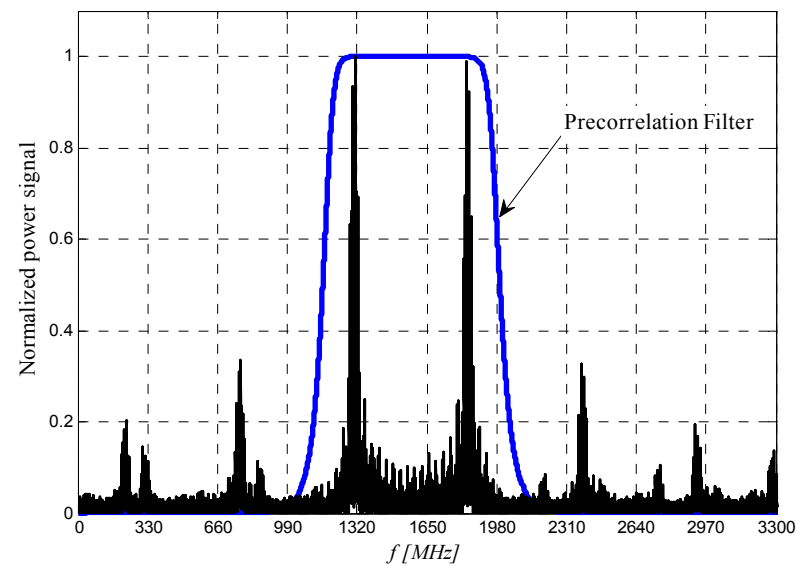

(a) $\quad \operatorname{BOCsin}(8,1)$ signal spectrum \& $24 \mathrm{MHz}$ bandlimited $\mathrm{P}-\mathrm{BW}$ filter.

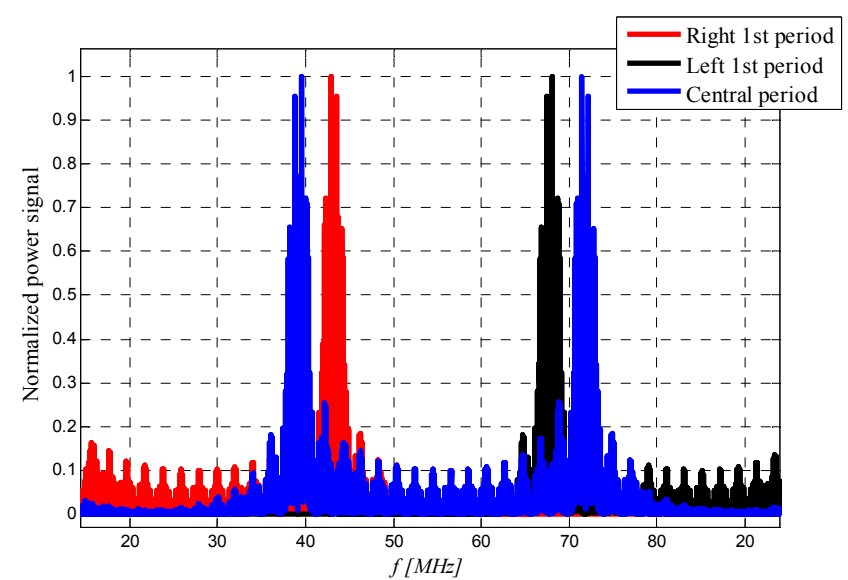

(b) Three periods of BOCsin $(8,1)$ signal spectrum after undersampling.

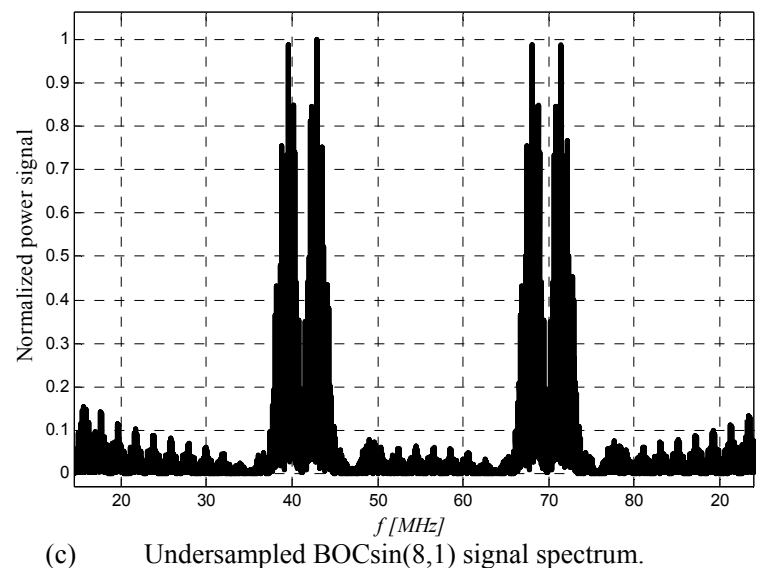

Fig. 5. BOCsin( 8,1$)$ signal spectrum before and after undersampling.

The first variant acquisition and tracking procedure, of our proposed method, consists of acquiring BOCsin $/ \cos (\alpha, \beta)$ modulated signals with $\alpha>\beta$, and more specifically with $\alpha>>\beta$, after undergoing a transformation based on undersampling process. The latter technique relies on sampling one desired signal with a frequency that is lower than twice the highest signal frequency (Shannon frequency) [51], [52], [53], [54]. The main purpose of such processing, which does not meet the Nyquist condition, is 


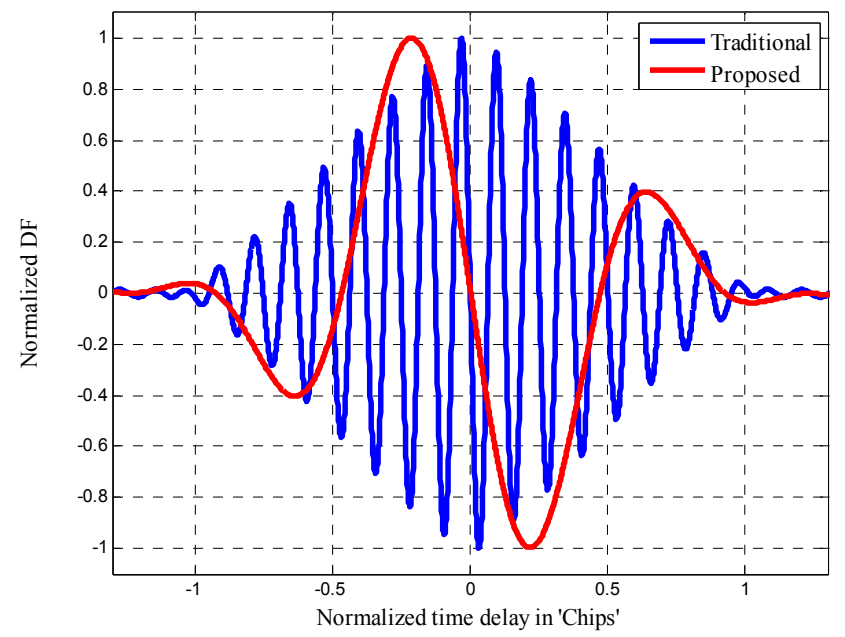

Fig. 6. DF of BOCsin $(8,1)$ modulated signal before and after using the first variant of the proposed method.

to bring closer the main lobes of $\mathrm{BOC} \sin / \cos (\alpha, \beta)$ signals and provide, thus signals similar to those of BOCsin $/ \cos (\alpha, \alpha)$. This operation allows, subsequently, the use of the classical $\operatorname{BOC}(\alpha, \alpha)$ or $\operatorname{CBCS}$ (third variant) or BOC-PRN receiving procedures to acquire this type of signals. Figure 5 shows an example of undersampling the BOCsin(8.1) signal.

Firstly, the received signal is limited to a bandwidth of $B=24 \mathrm{MHz}$ by the precorrelation (P-BW) bandpass filter as shown in Fig. 5(a). Then, the resulting signal, after conversion to the intermediate frequency $f_{\mathrm{IF}}$, is undersampled with a frequency that is equal to $f_{\mathrm{US}}=\left(f_{\mathrm{IF}}+B\right) / 3$ as shown in Fig. 5(b). Finally, the so obtained signal is processed by the acquisition and tracking stage to get the time delay estimation.

The DF corresponding to this first proposed variant for BOCsin $(8,1)$ modulated signal is shown in Fig. 6. As illustrated in this figure, the traditional method S-curve of BOCsin $(8,1)$ presents a large number of side zeroscrossings that create ambiguity in DLL tracking loop. Consequently, the tracking loop design for traditional $\operatorname{BOC} \sin (8,1)$ may be more problematic than that for BOCsin( $(1,1)$, especially with the conventional narrow correlator in the DLL. On the other hand, the same figure shows that BOCsin $(8,1) \mathrm{S}$-curve, for the proposed alternative, presents a very reduced number of side zeros-crossings compared to the classical $\mathrm{BOC} \sin (8,1)$ one. As a result, $\mathrm{BOC} \sin / \cos (\alpha, \beta)$ waveforms with high modulation orders can be processed using the proposed procedure combined with very simple acquisition and tracking structures similar to those used for BOCsin $/ \cos (\alpha, \alpha)$ modulated signals.

It should be noted, though, that this proposed variant is valid for both $\mathrm{BOC} \sin (\alpha, \beta)$ and $\mathrm{BOC} \cos (\alpha, \beta)$ modulated signals and its related transformation should be applied to both the received and locally generated signals. Regarding precorrelation filtering, several types of bandpass filters can be used such as coupled line filter, comb line filter, hairpin filter, stepped impedance filter, and so on. The precorrelation bandpass filter in the receiver plays the role

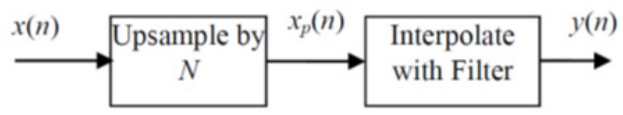

Fig. 7. Principle of sequence upsampling.

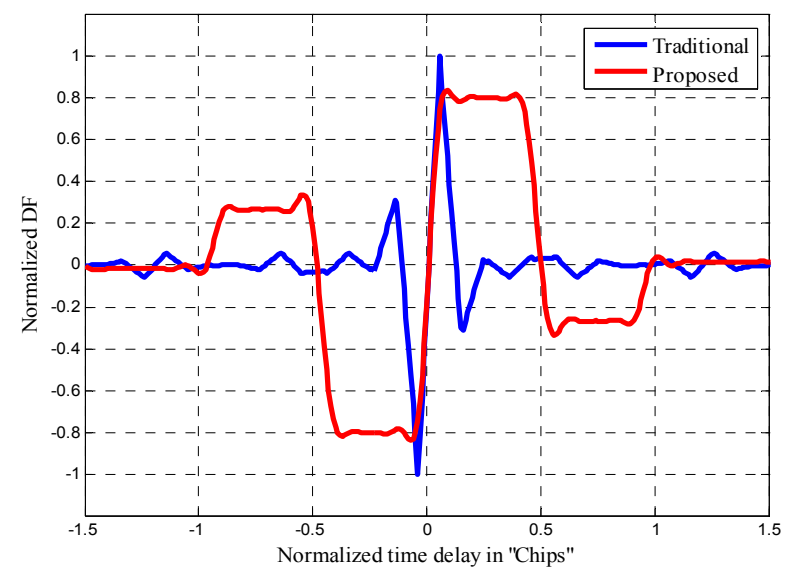

Fig. 8. DF corresponding to the second version, for $\operatorname{BOC}(5,5)$ modulated signal.

of anti-aliasing filter since it prevents undesired signals or noise to alias into the desired signal band.

The second reception alternative of our proposed method is based on time expansion or upsampling, which consists of scaling property that can be realized in " $Z$ ", domain [55]. The upsampling process, valid for BOCsin $/ \cos (\alpha, \beta)$ with $\alpha>1, \quad \beta>1$ and $\alpha=\beta$, and $\operatorname{BPSK}(\beta)$ signals, is shown in Fig. 7. Its principle consists firstly of inserting $N$ zeros between consecutive input samples of the input signal. After that, the resulting sequence is filtered leading to interpolation between the non-zero samples. Thus, the resulted signal creates the upsampled sequence. By using classical acquisition and tracking circuits, the latter is then correlated with its corresponding locally generated and upsampled sequence.

The S-curve corresponding to this second version, for BOCsin $(5,5)$ modulated signal, is shown in Fig. 8. As shown in Fig. 8, the absolute maxima of the second variant DF have approximately the same levels as those of the classical scheme DF. However, the second variant DF is more robust to synchronization/timing effects because its plateau is longer compared to the traditional one.

The third variant of our proposed scheme consists of acquiring more than five GNSS signals by a single local CBCS waveform replica generated by the GNSS receiver. The idea comes basically from the fact that $\operatorname{BOC} \sin (1,1)$, $\operatorname{CBOC}(6,1,1 / 11,+), \operatorname{TMBOC}(6,1,4 / 33)$ and $\operatorname{BOCcos}(1,1)$ are all very close to $\mathrm{CBCS}(20 \%)$ signal (See PSDs and ACFs in Fig. 1 and Fig. 3), which allows their acquisition by the latter one with a merely small power degradation. The resulting DFs of these different acquired signals are shown in Fig. 9. As illustrated in this figure, we observe that all the S-curves are very close except for small differences around the maxima and minima that do not influence the tracking process. Note that the procedure that is used herein is similar to those of step-chips and BOC-PRN structures [28], [56], [57]. 


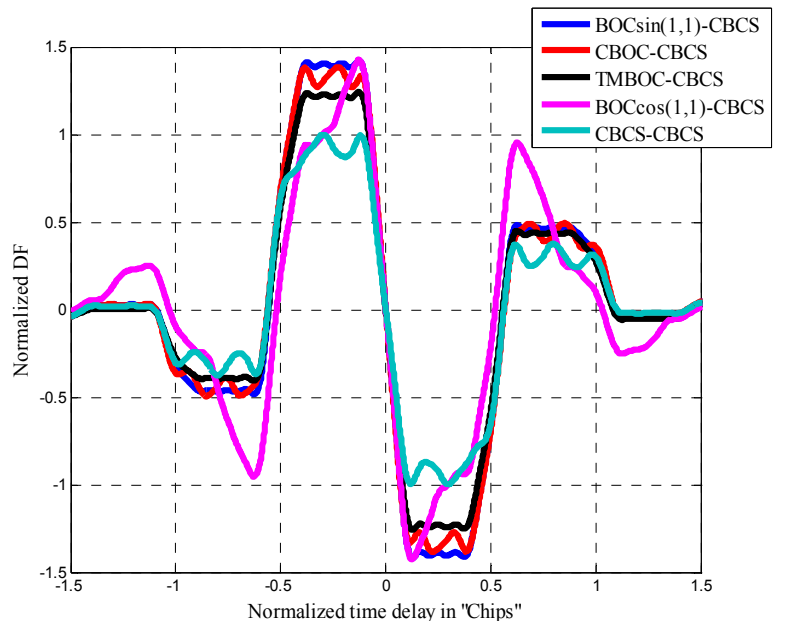

Fig. 9. $\mathrm{DFs}$ of $\operatorname{BOCsin}(1,1), \quad \mathrm{CBOC}(6,1,1 / 11,+)$, TMBOC $(6,1,4 / 33), \quad \operatorname{BOC} \cos (1,1)$ and $\operatorname{CBCS}(20 \%)$ acquired with $\mathrm{CBCS}(20 \%)$.

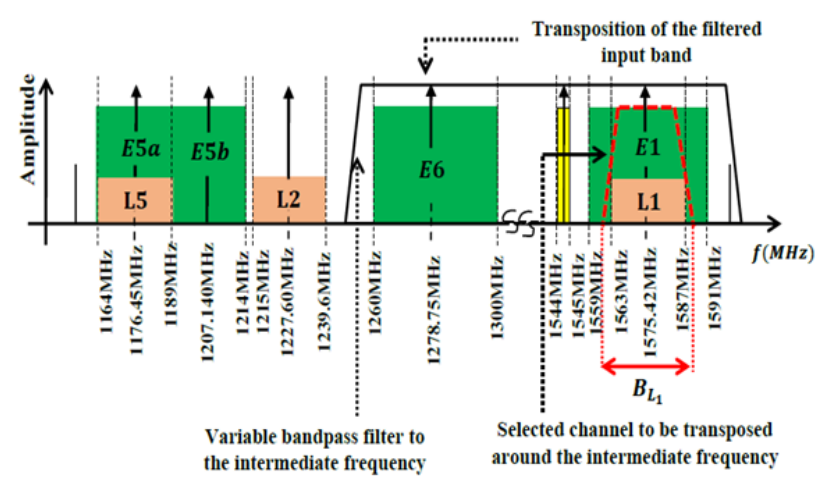

Fig. 10. Transposition of the input band and intermediate frequency filtering.

Figure 10 shows the process of implementing the input tape and the intermediate frequency filtering that is common to all three variants of our proposed method.

Initially, a primary selection of GNSS RF signals is performed using a broadband Radio Frequency (RF) bandpass filter, in order to recover the maximum possible of GNSS signals. Here, the filtered RF signal, via the GNSS receiver antenna, can be expressed as:

$$
\begin{aligned}
& S_{\mathrm{GNSS}}(t)= \\
& \sum_{i}^{k} \sqrt{P_{i}(t)} \cdot D_{i}\left(t-\tau_{i}\right) \cdot C_{i}\left(t-\tau_{i}\right) \cdot \cos \left[2 \pi\left(f_{p i}+f_{d i}\right) t+\phi_{i}\right] \\
& +W(t)
\end{aligned}
$$

where $P_{i}(t)$ is the $i^{\text {th }}$ signal received instantaneous power; $D_{i}(t)$ is the $i^{\text {th }}$ signal navigation data; $C_{i}(t)$ is the PRN code and subcarrier corresponding to the $i^{\text {th }}$ satellite; $\tau_{i}$ is the delay of the $i^{\text {th }}$ received signal; $f_{p_{i}}$ is the $i^{\text {th }}$ signal received carrier frequency; $f_{d_{i}}$ is the Doppler shift frequency corresponding to the $i^{\text {th }}$ satellite; $\phi_{i}$ is the $i^{\text {th }}$ received signal carrier phase; $W(t)$ is Additive White Gaussian Noise (AWGN).
Afterwards, as shown in Fig. 10, thanks to a variable bandpass filter, the channel to be converted into intermediate frequency $f_{\mathrm{IF}}$ is designated. In this case, $f_{\mathrm{C}}=$ $1575.42 \mathrm{MHz}$ is the selected GNSS L1 band center frequency and the desired $f_{\mathrm{IF}}$ is equal to $47.74 \mathrm{MHz}$. Hence, the IF signal can be written as [10]:

$$
S_{\mathrm{IF}}(t)=R\left\{S(t) \exp \left[\mathrm{j}\left(2 \pi f_{\mathrm{IF}} t+\phi(t)\right)\right]\right\}+b_{0}(t)
$$

where $\phi(t)$ now includes receiver clock instabilities and $b_{0}(t)$ is narrow band noise.

The channels selected for the first, second and third proposed method variants are given in Fig. 11 (a, b and c) respectively, and their characteristics are specified as follows:

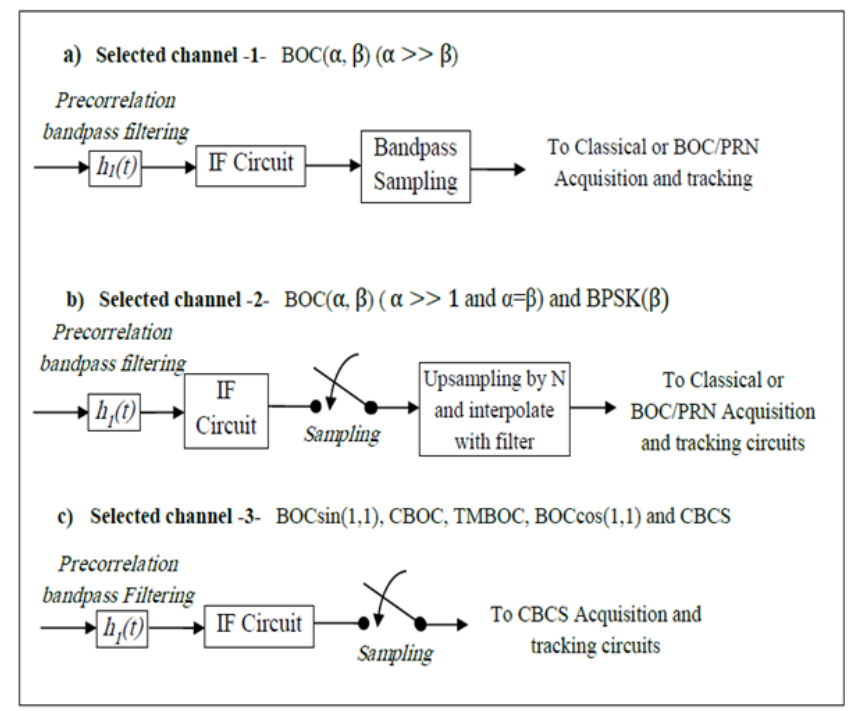

Fig. 11. Selected channels of the three proposed method variants.

\subsection{Channel-1}

Herein a $\mathrm{BOC} \sin / \cos (\alpha, \beta)$ modulated signal $(\alpha>>\beta)$ corresponding to the first category is filtered with a bandpass precorrelation filter with impulse response $h_{1}(t)$. The resulted signal is then centered on $f_{\mathrm{IF}}$ frequency. Afterwards, it is undersampled with a frequency that is equal to $f_{\mathrm{US}}=\left(f_{\mathrm{IF}}+B\right) / 3$ and then mixed with a local digital sinusoidal waveform and its 90-degree shifted version to get downconversion. Thus, the digitized signal can be given as [10]:

$S_{\mathrm{dig}, \mathrm{IF}}(k T)=R\left\{S(k T) \exp \left[\mathrm{j}\left(2 \pi f_{\mathrm{IF}} k T+\phi(k T)\right)\right]\right\}$

where $T$ is the sampling period that must be chosen to fulfill the undersampling process. The objective of digital down-conversion is to shift this latter component from the $f_{\mathrm{IF}}$ frequency down to baseband without losing phase information. The obtained baseband signal is given by [10]:

$$
S_{\text {base }}(k T)=R\{S(k T) \exp [\mathrm{j} \phi(k T)]\} .
$$


This signal is a complex quantity that can be written as a function of its in-phase $(I)$ and quadrature $(Q)$ components as follows [10]:

$$
S_{\text {base }}(k T)=R\left\{\left[S_{\mathrm{I}}(k T)+\mathrm{j} S_{\mathrm{Q}}(k T)\right] \exp [\mathrm{j} \phi(k T)]\right\}
$$

where $S_{\mathrm{I}}(k T)$ and $S_{\mathrm{Q}}(k T)$ are respectively the $I$ and $Q$ components of the baseband signal. $S_{\text {base }}(k T)$ is now correlated with a code replica that has undergone the same transformation. The resultant ACF is then processed by a DLL loop to get the DF.

Figure 12 shows the architecture of the classical acquisition and tracking circuits. As previously stated, this scheme enables the acquisition and tracking of $\mathrm{BOC} \sin / \cos (\alpha, \beta)$ modulated signals having undertaken the first version transformation and having been translated thus to baseband [10]. Note that the DLL is embedded with the Phase Locked Loop (PLL) structure whose discriminator output corresponds to the phase error between the received signal and the carrier replica. The DLL may generate either a $\mathrm{BOC} \sin / \cos (\alpha, \beta)$ or a $\mathrm{CBCS}$ code replica and uses it for tracking the phase of the received code.

\subsection{Channel-2}

The received signal here is $\mathrm{BOC} \sin / \cos (\alpha, \alpha)$ or $\operatorname{BPSK}(\beta)$ modulated $(\alpha>1)$, corresponding to the second category. Similarly to the first channel, the received signal is filtered with the same bandpass precorrelation filter $h_{1}(t)$ as that of channel one. The obtained signal is then centered on $f_{\mathrm{IF}}$ frequency and upsampled. The latter operation is followed by an adequate interpolation. Finally, the resultant signal, after digital down-conversion, is acquired and tracked using the same classical acquisition and tracking architecture as that shown in Fig. 12. In this scheme, for $\operatorname{BOC} \sin / \cos (\alpha, \alpha)$ and $\operatorname{BPSK}(\beta)$ received signals, the locally generated subcarrier can be $\mathrm{BOC} \sin / \cos (\alpha, \alpha)$ or $\mathrm{CBCS}$, or PRN code.

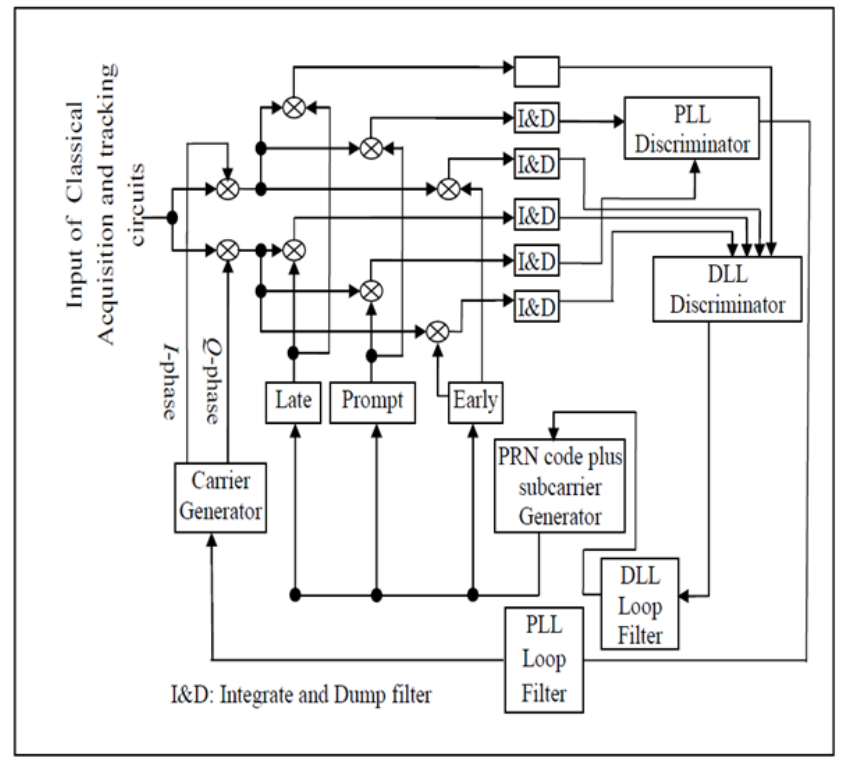

Fig. 12. Classical acquisition and tracking circuits.

\subsection{Channel-3}

Channel-3 is conceived to receive $\mathrm{BOC} \sin / \cos (1,1)$, TMBOC, CBOC, and CBCS signals. After filtering and sampling operations, the resulting signal is sent directly to the signal processing blocks. Note that for this third proposed method variant, the acquisition circuit is equivalent to that of Fig. 12 except for the code and subcarrier generator which is herein confined to CBCS subcarrier generation to acquire and track all the four sub mentioned channel-3 signals. In other words, the unique generator that must be used in the acquisition circuit is that of CBCS.

\section{Simulation Results}

In this section, the performances in terms of MP mitigation and noise effect reduction of the three proposed variants are firstly evaluated by using, respectively, the running average errors and the Root Mean Square Error (RMSE) criteria. At the meantime, a comparative study is conducted between the proposed variants and their classical counterparts.

The running average error is calculated by using the maximum error resulting from the presence of one single MP with a certain phase, delay and amplitude. It is worth noting that the computation of the MP-induced code tracking error envelope consists of finding the point, where the discriminator output crosses the origin. This point represents the point where the DLL will lock. In all simulations, the MP signal is taken with amplitude of 0.5 (which corresponds to the usual value). The MP delay variation range in reality must be chosen considering the PRN correlation characteristics that cause the filtering, by the DLL loop, of all the MPs with delays greater than 1.5 chips (approximately 440 meters) with respect to the Line Of Sight (LOS). However, in order to see more clearly the test results around higher MP delays, the MP delay variation range, in all simulations, is chosen from 0 to 1320 meters. The MP error envelopes correspond to the maximum values obtained when the MP signal is at $0^{\circ}$ "in phase" or $180^{\circ}$ "out of phase" with respect to the LOS [58]. Once the MP error is calculated, the absolute envelope values and their cumulative sum are determined, with the same norm as that in [58], to get the MP running average error.

The RMSE is used versus SNR that varies from $-40 \mathrm{~dB}$ to $-20 \mathrm{~dB}$. The choice of the two latter boundaries permits the support of both very weak signals and those with an adequate SNR ratio. The SNR here is defined as the $\mathrm{C} / \mathrm{N}_{0}$ ratio divided by the $\mathrm{RF}$ signal bandwidth. It is worth noting that the computation of the RMSE induced code tracking estimation bias consists of finding also (in noisy situation) the point where the DLL discriminator output is null.

\subsection{The First Proposed Variant}

The scheme of undersampling is implemented together with the classical one. A $36 \mathrm{MHz}$ P-BW receiver 


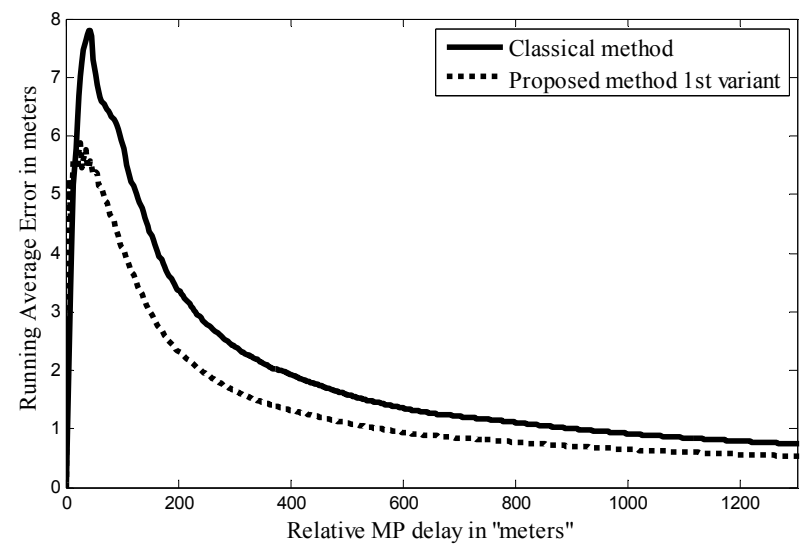

Fig. 13. Running average errors of the classical and proposed first variant reception of $\operatorname{BOC} \cos (15,2.5)$ modulated signal.

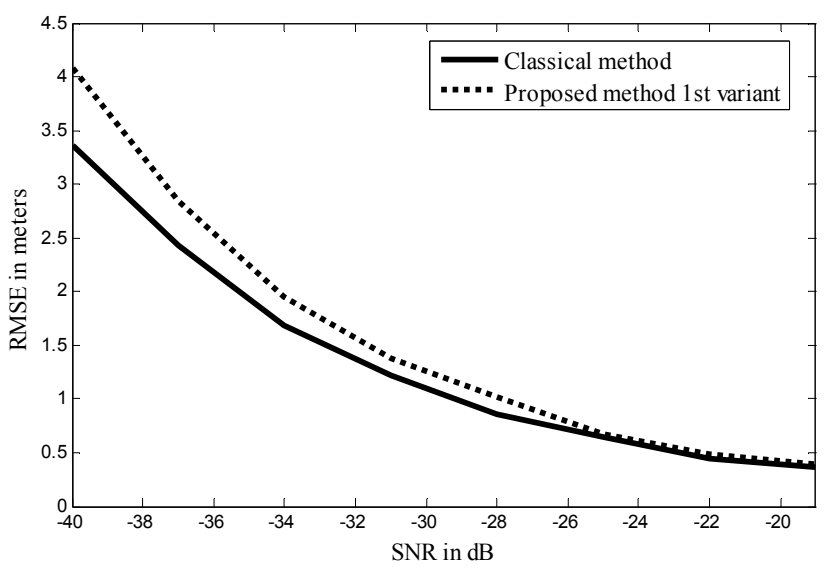

Fig. 14. RMSEs of the classical and proposed first variant reception of $\mathrm{BOC} \cos (15,2.5)$ modulated signal.

bandpass filter is used and a Galileo BOCcos $(15,2.5)$ signal is chosen to be received and transformed into BOCcos $(2.5,2.5)$. Here, the early late chip spacing between the DLL correlators is taken equal to $T_{\mathrm{C}} / 5$. The running average errors results are shown in Fig. 13. As illustrated in this figure, the proposed method presents the overall best performances in terms of MP mitigation because it is only sensitive for short MP delays.

Concerning the noise effect, as illustrated in Fig. 14, as soon as the SNR value approaches $-25 \mathrm{~dB}$ (which represents an average level of SNR), the RMSE of the proposed method approaches that of the classical one, which proves the applicability of the first proposed variant. The RMSE difference, between both schemes (which has a maximum value of 0.75 meter for the minimum value of SNR) is due to the spectrum overlapping.

\subsection{The Second Proposed Variant}

Herein, the reception of GPS BPSK(10) signal is chosen using a $24 \mathrm{MHz}$ (corresponding to the radionavigation satellite service bandwidths) P-BW receiver bandpass filter. After transformation, the resulting signal is similar to that of BPSK(1). Here, the early late chip spacing between the DLL correlators is also chosen equal to $T_{\mathrm{C}} / 5$. The re-

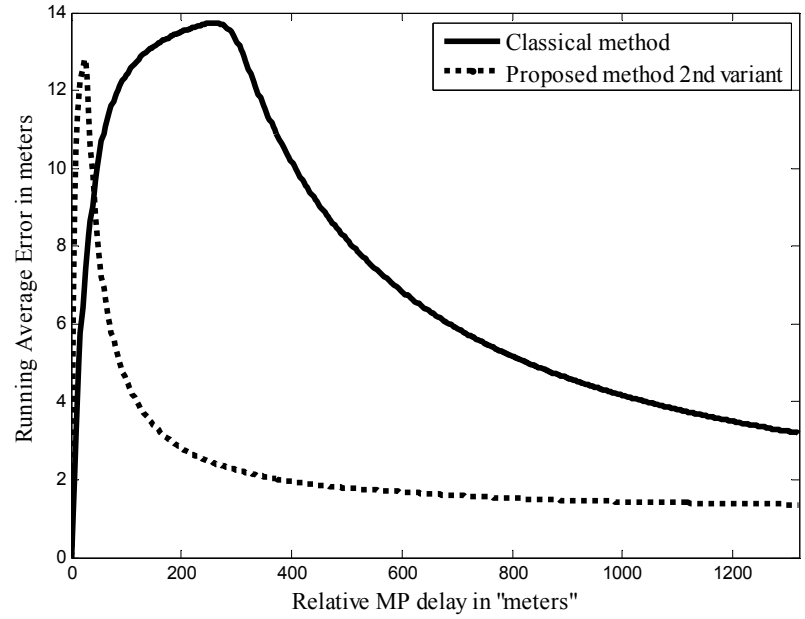

Fig. 15. Running average errors of the classical and proposed second variant reception of $\operatorname{BPSK}(10)$ modulated signal.

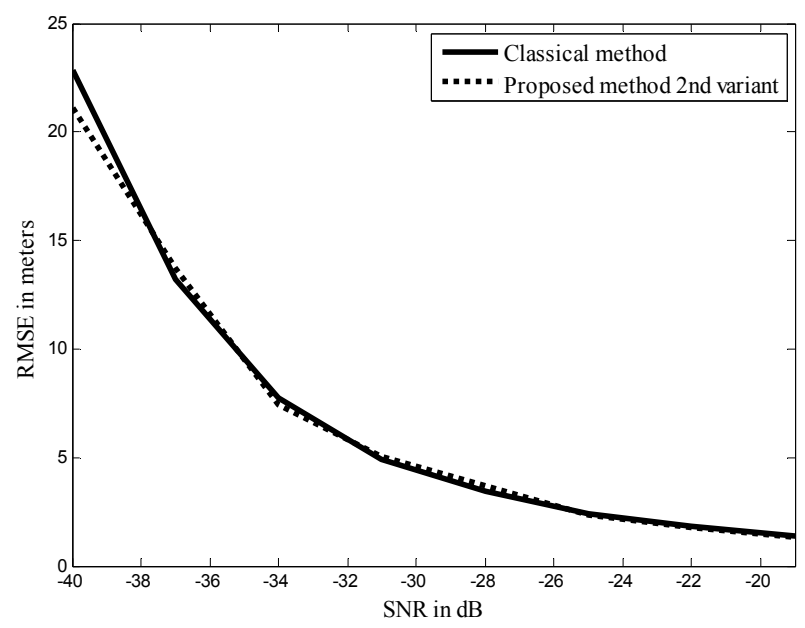

Fig. 16. RMSEs of the classical and proposed second variant of BPSK(10) modulated signal.

sults corresponding to the running average errors and the RMSE are shown in Fig. 15 and Fig. 16, respectively.

The running average error curves in Fig. 15 show a big difference between the classical method and the proposed one. In fact, the MPs effect on our proposed method is almost totally eliminated except for low values of the delay to which it remains sensitive. This is because the transformation of the proposed second variant retains the same properties of the original signals. For RMSE metric, Figure 16 shows that the proposed second variant presents the same performance as that of the classical method in terms of resistance to the noise for all SNR values.

\subsection{The Third Proposed Variant}

Herein, the $\mathrm{CBCS}$ signal has been used to receive separately BOCsin/ $\cos (1,1), \mathrm{CBOC}, \mathrm{CBCS}$ and TMBOC, modulated signals. At this time, the early late chip spacing between the DLL correlators is selected equal to $T_{\mathrm{C}} / 5$ for BOCsin $(1,1), \mathrm{CBOC}, \mathrm{CBCS}$ and TMBOC signals and $T_{\mathrm{C}} / 2$ for $\mathrm{BOC} \cos (1,1)$ one. Note that the value of $T_{\mathrm{C}} / 2$ is chosen because it does not provoke any ambiguity problem in the 


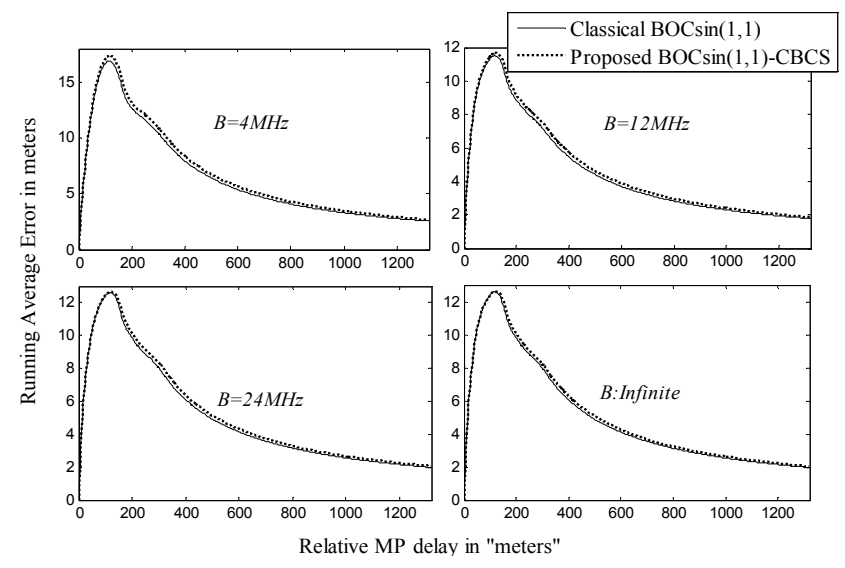

Fig. 17. Running average errors of the classical and proposed third variant reception of $\operatorname{BOCsin}(1,1)$ modulated signal for different P-BWs.

DLL. In Fig. 17, Fig. 18, Fig. 19 and Fig. 20, the results of the running average errors of both the proposed and classical schemes are represented, for P-BW bandwidths of $4 \mathrm{MHz}, 12 \mathrm{MHz}, 24 \mathrm{MHz}$ and infinity, respectively.

Conferring to Fig. 17, concerning BOCsin(1,1) received signal case, the running average error values for each of the classical schemes, corresponding to P-BWs of $4 \mathrm{MHz}, 12 \mathrm{MHz}, 24 \mathrm{MHz}$ and infinity, are approximately equal to those of the proposed third variant. In fact, the maximal differences between the curves' results of both schemes for all P-BWs do not exceed 0.344 meter, which qualifies the proposed third version MP performance to be very close to that of the classical case and confirming, thus, its efficiency for BOCsin signal reception.

According to Fig. 18, related to $\mathrm{CBOC}$ signal reception case, the running average error values for each of the classical schemes, corresponding to P-BWs of $4 \mathrm{MHz}$, $12 \mathrm{MHz}, 24 \mathrm{MHz}$ and infinity, compared to those of the proposed third variant, are initially the same over the range of small MP delays (few meters). Then, for the $4 \mathrm{MHz}$ PBW case, both the proposed and classical curves are approximately superposed. In the meantime, for all the remaining P-BWs $12 \mathrm{MHz}, 24 \mathrm{MHz}$ and infinity, the values of the proposed third variant running average error values become lower over the entire MP delays range and decay more rapidly to zero than those of classical schemes. Besides, the gap between the proposed and classical schemes, for all P-BWs of $12 \mathrm{MHz}, 24 \mathrm{MHz}$ and infinity, increases when the P-BW is augmented reaching the maximal differences values of 1.64 meters, 3.03 meters and 3.06 meters, respectively.

In summary, according to these results, for CBOC reception in MP environment, the proposed scheme presents performance that is very close to the classical one for $4 \mathrm{MHz}$ P-BW, while it performs better than the classical one for $12 \mathrm{MHz}, 24 \mathrm{MHz}$ and infinity P-BWs, and improves as $\mathrm{P}-\mathrm{BW}$ increases.

From Fig. 19, corresponding to TMBOC signal, the running average error values of each of the classical schemes corresponding to P-BWs of $4 \mathrm{MHz}, 12 \mathrm{MHz}$, $24 \mathrm{MHz}$ and infinity, compared to those of the proposed

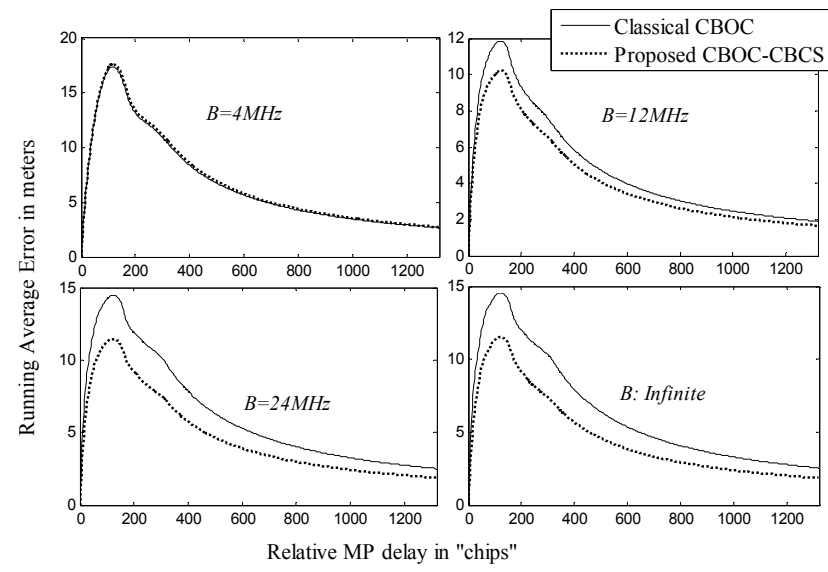

Fig. 18. Running average errors of the classical and proposed third variant reception of $\mathrm{CBOC}$ modulated signal for different P-BWs

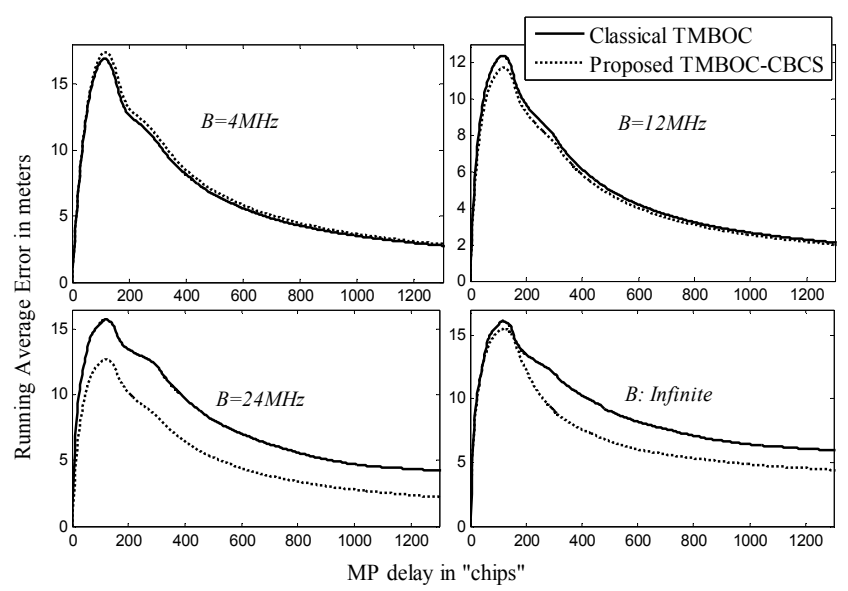

Fig. 19. Running average errors of the classical and proposed third variant reception of TMBOC modulated signal for different P-BWs.

third variant, are initially the same over the range of small MP delays (few meters). Then, for the whole remaining MP delays range, the $4 \mathrm{MHz}$ error values of the proposed variant are greater than those of the classical one, with a maximal reached gap not exceeding 0.82 meter.

At the same time, except for the $4 \mathrm{MHz}$ P-BW case, the values of the proposed variant for all other P-BWs become lower and decay more rapidly to zero than those of classical schemes. Moreover, the gaps between the proposed and classical schemes curves, for P-BWs of $12 \mathrm{MHz}, 24 \mathrm{MHz}$ and infinity, increase when the P-BW is increased reaching their maximal values of approximately 0.66 meter, 2.9 meters and 0.58 meters, respectively. According to these results, for TMBOC reception in MP environment, the proposed scheme presents performance that is less good but close to the classical one for the $4 \mathrm{MHz}$ P-BW; while, for the $12 \mathrm{MHz}, 24 \mathrm{MHz}$ and infinity $\mathrm{P}-\mathrm{BWs}$, it is always the best and improves as P-BW increases. Hence, TMBOC reception via the proposed third variant in MP environment is efficient.

In Fig. 20, for the $\mathrm{BOC} \cos (1,1)$ reception case, the running average error values for each of the classical and proposed schemes, corresponding to P-BWs of $4 \mathrm{MHz}$, 


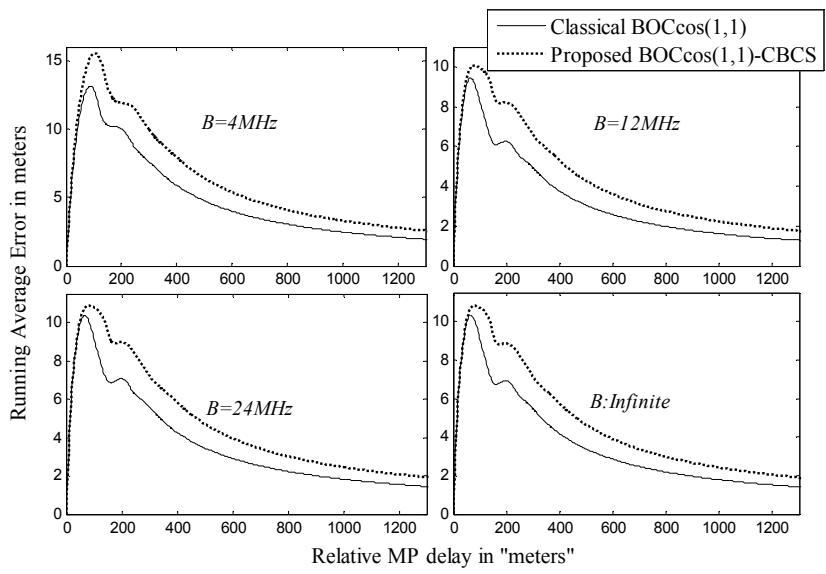

Fig. 20. Running average errors of the classical and proposed third variant reception of $\mathrm{BOC} \cos (1,1)$ modulated signal for different P-BWs.

$12 \mathrm{MHz}, 24 \mathrm{MHz}$ and infinity are represented. A comparative study shows that, in this case, for all P-BWs, the proposed and classical methods curves are the same only for short MP delays. Then, for delays greater than approximately 100 meters, the obtained curves values of the proposed method are, always and for all P-BWs, greater than those of the classical ones. However, the gaps maximal values between the proposed and classical schemes curves, for all considered P-BWs, are situated between 1 and 2.47 meters. Regarding the advantages provided by the proposed third variant, such a degradation is acceptable and qualifies it to be efficient for $\mathrm{MC} / \mathrm{MF}$ reception in in $\mathrm{MP}$ environment.

The code tracking RMSEs of all the above mentioned classical and proposed schemes reception of BOCsin, CBOC, TMBOC and BOCcos signals, respectively, for four different values of the P-BW (4 MHZ, $12 \mathrm{MHz}$, $24 \mathrm{MHz}$, and infinity) are illustrated in Fig. 21, Fig. 22, Fig. 23 and Fig. 24.

According to Fig. 21, dedicated to BOCsin $(1,1)$ reception, the proposed scheme RMSE curves corresponding to P-BWs of $4 \mathrm{MHz}, 12 \mathrm{MHz}, 24 \mathrm{MHz}$ and infinity, compared to those of the classical ones, are practically the same, except for SNR less than $-32 \mathrm{~dB}$. In this case, the proposed third version becomes less performant compared to the classical method due to a small degradation that does not exceed, for all considered P-BWs, 0.475 meters (for infinite P-BW, at $-40 \mathrm{~dB}$ ), which proves the efficiency of the proposed third variant in noisy environment.

According to Fig. 22, dedicated to CBOC reception, the RMSE curves values for each of the proposed third method schemes, corresponding to all considered P-BWs, are less than those of the classical ones over the entire SNR range. In addition, as the P-BW increases, the better is the performance of the proposed variant compared to the classical scheme. Therefore, CBOC reception with the proposed third variant presents a better resistance to the noise compared to the classical scheme.

From Fig. 23, dedicated to TMBOC reception, the RMSE curves values of the proposed method correspond-

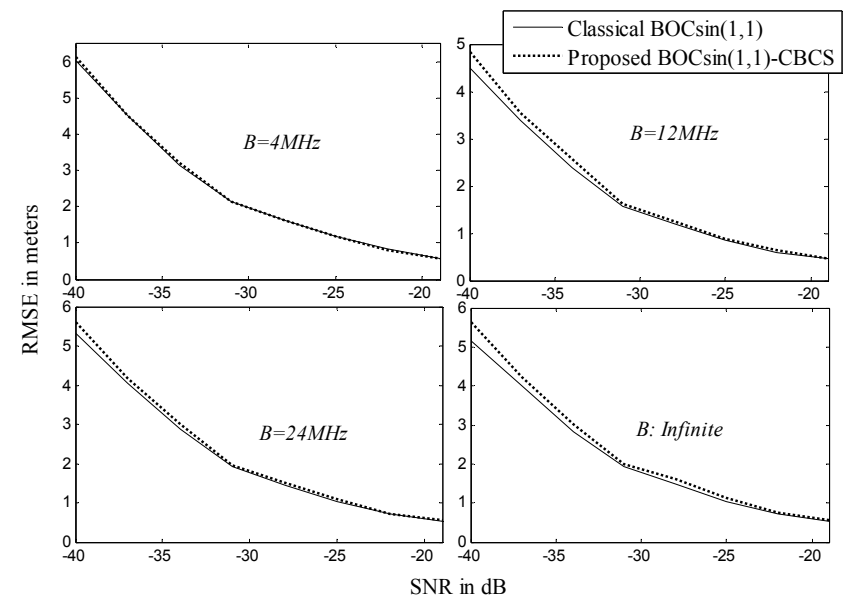

Fig. 21. RMSEs of the classical and proposed third variant reception of $\operatorname{BOC} \sin (1,1)$ modulated signal for different P-BWs.

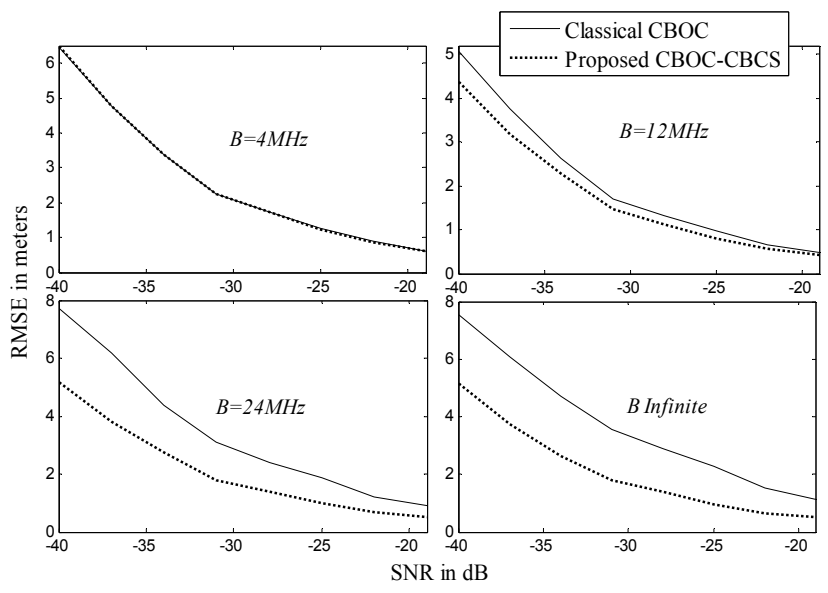

Fig. 22. RMSEs of the classical and proposed third variant reception of $\mathrm{CBOC}$ modulated signal for different P-BWs.

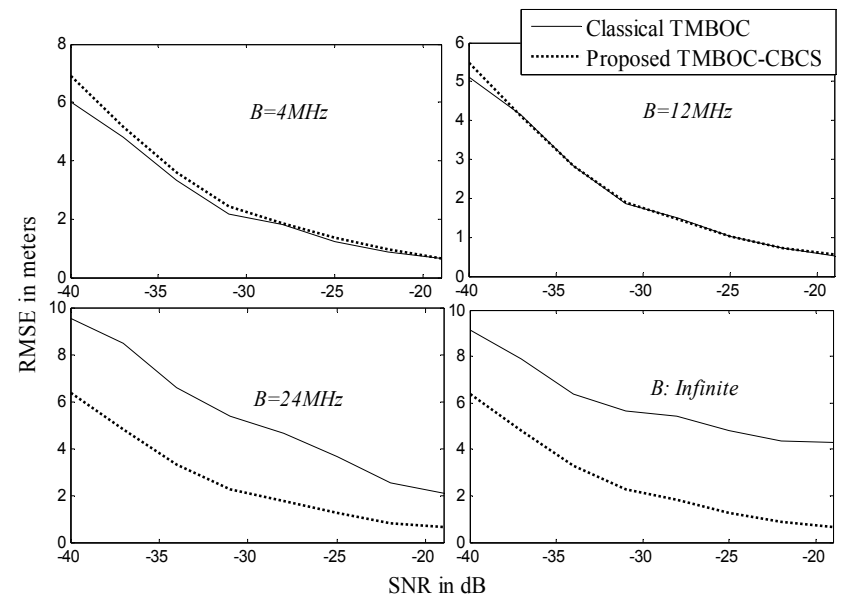

Fig. 23. RMSEs of the classical and proposed third variant reception of TMBOC modulated signal for different P-BWs.

ing to $4 \mathrm{MHz}$ and $12 \mathrm{MHz}$ P-BWs are approximatively the same as those of the classical scheme in the SNR range $[-37 \mathrm{~dB},-20 \mathrm{~dB}]$ and present an insignificant degradation. 


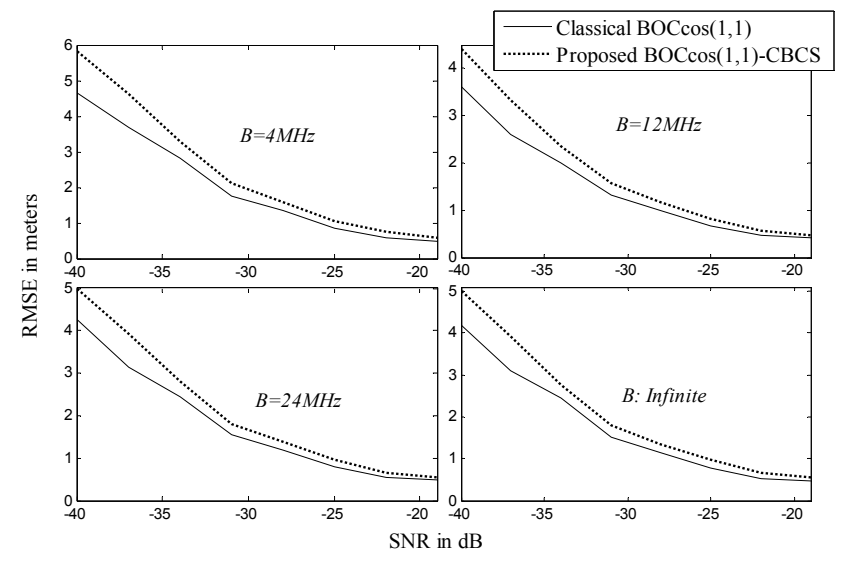

Fig. 24. RMSEs of the classical and proposed third variant reception of $\mathrm{BOC} \cos (1,1)$ modulated signal for different P-BWs.

However, the proposed third variant for P-BWs of $24 \mathrm{MHz}$ and infinity, performs better than the classical method reaching improvement between 2 meters and 4 meters over the entire SNR range and as the P-BW increases the better is the performance of the proposed variant compared to the classical scheme. Therefore, the TMBOC reception with the proposed third variant presents better performances in comparison to that with the classical scheme.

Figure 24, dedicated to BOCcos reception, shows that the RMSE curves values for each of the proposed third variant, corresponding to all P-BWs, are a little higher than those of the classical ones over the entire SNR range. It should be noted, however, that for SNR values greater than $-35 \mathrm{~dB}$, the RMSE curves gap values does not exceed 0.5 meters. Thus, BOCcos signals reception with the proposed third variant, compared to the classical scheme reception, is also efficient in a noisy environment.

In summary, the simulation results of the third proposed variant concerning both running average errors and RMSE criteria qualify the CBCS signal to be adopted successfully by $\mathrm{MC} / \mathrm{MF}$ reception of $\mathrm{BOC} \sin / \cos (1,1)$, CBOC and TMBOC modulated signals in MP and noisy environments and under conditions where the received signals are not identified or known in advance.

\subsection{MC/MF Error Reduction in Degraded Environment}

Finally, in this part, a last test is conducted to study the proposed method performance as a function of the number of combined MC/MF (GPS, Galileo and GLONASS) signals of Satellites In Space (SIS). For this purpose, we assume a neglected offset between different GNSS systems time scales. The geometry of simulated satellites has been realized according to a predefined placement taking into account the noise and MP environmental factor. In this topology, satellites are placed randomly since the regular case does not reflect the real-world conditions.

\begin{tabular}{|c|c|c|c|c|}
\hline & $\begin{array}{c}\text { Initial } \\
\text { error in } \\
\text { meters }\end{array}$ & \multicolumn{3}{|c|}{ Error reduction in \% } \\
\hline $\begin{array}{c}\text { Mean of } \\
\text { TOA } \\
\text { RMSE in } \\
\text { meters }\end{array}$ & $\begin{array}{c}4 \text { GPS } \\
\text { visible } \\
\text { SIS }\end{array}$ & $\begin{array}{c}2 \text { GPS + 2 } \\
\text { Galileo } \\
\text { visible SIS }\end{array}$ & $\begin{array}{c}3 \text { GPS + } \\
\text { Galileo } \\
\text { visible SIS }\end{array}$ & $\begin{array}{c}3 \text { GPS + 1 } \\
\text { Galileo + } 1 \\
\text { GLONASS } \\
\text { visible SIS }\end{array}$ \\
\hline 0.5 & 0.5694 & $24 \%$ & $30 \%$ & $40 \%$ \\
\hline 3.0 & 1.9667 & $25 \%$ & $30 \%$ & $41 \%$ \\
\hline 5.5 & 3.6213 & $27 \%$ & $31 \%$ & $42 \%$ \\
\hline 8.0 & 5.2755 & $27 \%$ & $30 \%$ & $41 \%$ \\
\hline 10.5 & 6.9212 & $27 \%$ & $31 \%$ & $42 \%$ \\
\hline 13.0 & 8.5343 & $26 \%$ & $31 \%$ & $41 \%$ \\
\hline 15.5 & 10.1893 & $26 \%$ & $31 \%$ & $42 \%$ \\
\hline 16.5 & 10.2003 & $26 \%$ & $31 \%$ & $42 \%$ \\
\hline
\end{tabular}

Tab. 1. Percentage error reduction as a function of the TOA RMSE.

The receiver selects, among the MC/MF visible SISs signals, those whose SNRs are the best possible. Among the different possibilities of this selection process, four cases of interest are chosen. In the first one, four signals are selected appertaining all to the same GPS system constellation and representing, thus, the standalone GPS reception. In the second case, the two first case GPS SISs having the lowest SNR levels are replaced by two Galileo SISs with higher SNR levels. In the third case, the same SISs used in the second case are reinforced by one more selected GPS SIS, increasing, thus, the number of selected SISs to five. In the last case, the third case Galileo SIS having the lowest SNR level is replaced by one MF GLONASS SIS with higher SNR level. The selected SISs in each of the four cases are used to perform the pseudo-range measurements with a given RMSE of Time Of Arrival (TOA) estimation. The latter one varies, for each SIS, from 0 to 16.5 meters. The upper value of this interval corresponds to the mean of the maximum errors reached by the three proposed variants. In this simulation, the receiver position estimation, based on the least square method, is repeated according to 1000 iterations. The results, in terms of percentage error reduction, as a function of the RMSE, are illustrated in Tab. 1.

As shown in Tab. 1, the increase in the number of different multi-constellations SISs implies a reduction of the average error and consequently an improvement of the performances. In fact, percentage error reduction varies from $24 \%$ (for four selected SISs corresponding to GPS and Galileo) to $42 \%$ for an optimal combination of the three systems, i.e. GPS, Galileo and GLONASS. The latter situation proves that the MC/MF systems provide a best improvement compared to the standalone GPS.

\section{Conclusion}

In this paper, an efficient method for receiving a diversity of GNSS signals in degraded environments is proposed through three variants, each using a specific transformation adapted to a particular type of signals and combined with a single classical receiver structure. This method offers less complexity and thus lower cost con- 
cerning the overall receiver scheme. On the other hand, the proposed versions bring an efficient solution to weak power signals problem and benefit naturally from all advantages acquired by the PVT calculator thanks to the MC/MF-GNSS receiver design. The simulation results showed that the three proposed variants have satisfactory performances and most often better, in terms of MP mitigation and noise resistance, compared to the classical schemes that are not yet able to determine any position due to the use of a single GNSS system satellite constellation. In addition, they work for short/long weak/strong LOS or MP signals and achieve better performances than the traditional scheme for all MP signal delays, amplitudes and phases and for any P-BW of the receiver filter.

\section{Acknowledgments}

The authors would like to thank Madame Emilie Le Baillif, who carefully read the revised version of manuscript and made useful and detailed comments.

\section{References}

[1] KAPLAN, E. D., HEGARTY, C. J. Understanding GPS: Principles and Applications. $2^{\text {nd }}$ ed., rev. London (UK): Artech House, 2006. ISBN: 9781580538947

[2] BETZ, J. W. Binary offset carrier modulations for radionavigation. Navigation, Journal of the Institute of Navigation, 2002, vol. 48, no. 4, p. 227-246. DOI: 10.1002/j.2161-4296.2001.tb00247.x

[3] HEIN, G. W., AVILA-RODRIGUEZ, J. A., WALLNER, S., et al. MBOC: The new optimized spreading modulation recommended for Galileo L1 OS and GPS L1C. Inside GNSS, 2006, vol. 1, no. 4, p. 57-65.

[4] AVILA-ROdRIGUEZ, J. A., WALLNER, S., HEIN, G. W., et al. CBOC: An implementation of MBOC. In CNES-ESA, 1st Workshop on GALILEO Signals and Signal Processing. Toulouse (France), 2006. $10 \mathrm{p}$.

[5] AVILA-RODRIGUEZ, J. A. On Generalized Signal Waveforms for Satellite Navigation. Ph.D. Dissertation. University FAF Munich, 2008.

[6] HEGARTY, C., BETZ, J. W., SAIDI, A. Binary coded symbol modulations for GNSS. In Proceedings of the 60th Annual Meeting of the Institute of Navigation. Dayton (OH, USA), 2004, p. 56-64.

[7] HEIN, G. W., AVILA-RODRIGUEZ, J. A., RIES, L., et al. A candidate for the Galileo L1 OS optimized signal. In Proceedings of the 18th International Technical Meeting of the Satellite Division of the Institute of Navigation (ION GNSS 2005). Long Beach (CA, USA), 2005, p. 833-845.

[8] FLISSI, M., ROUABAH, K., CHIKOUCHE, D., et al. Performance of new BOC-AW-modulated signals for GNSS system. Eurasip Journal of Wireless Communications and Networking, 2013 , no. 124,18 p. DOI: $10.1186 / 1687-1499-2013-124$

[9] FLISSI, M., ROUABAH, K., ATIA, S., et al. Consistent BCS modulated signals for GNSS applications. IET Signal Processing, 2017, vol. 11, no. 4, p. 415-421, DOI: 10.1049/iet-spr.2016.0200

[10] PARKINSON, B. W., SPILKER, J. J., AXELRAD, P., ENGE, P. Global Positioning System: Theory and Applications. $1^{\text {st }}$ ed.
American Institute of Aeronautics and Astronautics, 1996, vol. 1, p. 793. ISBN: $1-56347-106-X$

[11] RAMA KRISHNA RAO, B., SARMA, A. D., RAVI KUMAR, Y. Technique to reduce multipath GPS signals. Current Science, 2006, vol. 90 , no. 2 , p. 207-211.

[12] CHUNG, L. C., JUANG, J. C. An adaptive multipath mitigation filter for GNSS applications. Eurasip Journal on Advances in Signal Processing, 2008, 10 p. DOI: 10.1155/2008/214815

[13] IRSIGLER, M., EISSFELLER, B. Comparison of multipath mitigation techniques with consideration of future signal structures. In Proceedings of the 16th International Technical Meeting of the Satellite Division of the Institute of Navigation (ION GPS/GNSS 2003). Portland (OR, USA), 2003, p. 2584-2592.

[14] VAN DIERENDONCK, A. J., FENTON, P., FORD, T. Theory and performance of narrow correlator spacing in a GPS receiver. Navigation: Journal of the Institute of Navigation, 1992. vol. 39, no. 3, p. 265-283. DOI: 10.1002/j.2161-4296.1992.tb02276.x

[15] Rouabah, K., ChiKouche, D., Bouttout, F., et al. GPS/Galileo multipath mitigation using the first side peak of double delta correlator. Wireless Personal Communications, 2011, vol. 60, no. 2, p. 321-333. DOI: 10.1007/s11277-010-9946-2

[16] SAHMOUDI, M., LANDRY, R. J. Multipath mitigation techniques using maximum-likelihood principle. Inside GNSS, 2008 , vol. 3 , no. 8, p. 24-29.

[17] VAN NEE, R. SIEREVELD, J. FENTON, P., TOWNSEND, B. The multipath estimating delay lock loop: approaching theoretical accuracy limits. In Proceedings of the IEEE Position Location and Navigation Symposium. Las Vegas (NV, USA), 1994, p. 246-251. DOI: 10.1109/PLANS.1994.303320

[18] HU, Y., SONG, M., MENG, B., et al. An efficient method for GPS multipath mitigation using the Teager-Kaiser-operator-based MEDLL. Radioengineering, 2013, vol. 22, no. 4, p. 1202-1210.

[19] SAHMOUDI, M., AMIN, M. G. Fast Iterative MaximumLikelihood Algorithm (FIMLA) for multipath mitigation in the next generation of GNSS receivers. IEEE Transactions on Wireless Communications, 2008, vol. 7, no. 11, p. 4362-4374. DOI: 10.1109/T-WC.2008.070700

[20] ZHANG, Z., LAW, C. L. Short-delay multipath mitigation technique based on virtual multipath. IEEE Antennas and Wireless Propagation Letters, 2005, vol. 4, p. 344-348. DOI: 10.1109/LAWP.2005.857038

[21] NUNES, F. D., SOUSA, F. M. G., LEITAO, J. M. N. Gating functions for multipath mitigation in GNSS BOC signals. IEEE Transactions on Aerospace and Electronic Systems, 2007 vol. 43, no. 3, p. 951-964. DOI: 10.1109/TAES.2007.4383585

[22] JULIEN, O., MACABIAU, C., CANNON, M. E., et al. ASPeCT: unambiguous sine-BOC(n,n) acquisition/tracking technique for navigation applications. IEEE Transactions on Aerospace and Electronic Systems, 2007, vol. 43, no. 1, p. 150-162. DOI: 10.1109/TAES.2007.357123

[23] BURIAN, A., LOHAN, E. S., RENFORS, M. K. Efficient delay tracking methods with sidelobes cancellation for BOC modulated signals. Eurasip Journal on Wireless Communications and Networking, 2007, 20 p. DOI: 10.1155/2007/72626

[24] YAO, Z., LU, M., FENG, Z. Unambiguous technique for multiplexed binary offset carrier modulated signals tracking. IEEE Signal Processing Letters, 2009, vol. 16, no. 7, p. 608-611. DOI: 10.1109/LSP.2009.2020462

[25] CHEN, H., REN, J., JIA, W., et al. Simultaneous perturbation stochastic approximation for unambiguous acquisition in CosineBOC signals. Radioengineering, 2013, vol. 22, no. 2, p. 578-585.

[26] ATTIA, S., ROUABAH, K., CHIKOUCHE, D., et al. Side peak cancellation method for sine-BOC $(m, n)$-modulated GNSS signals. 
Eurasip Journal on Wireless Communications and Networking, 2014, vol. 34, 14 p. DOI: 10.1186/1687-1499-2014-34

[27] REN, J., YANG, G., JIA, W. M., YAO, M. Unambiguous tracking method based on combined correlation functions for sine/cosine$\mathrm{BOC}, \mathrm{CBOC}$ and AltBOC modulated signals. Radioengineering, 2014, vol. 23, no. 1, p. 244-251.

[28] DOVIS, F., MULASSANO, P., PRESTI, L. L. A novel algorithm for the code tracking of $\operatorname{BOC}(n, n)$ modulated signals. In Proceedings of the 18th International Technical Meeting of the Satellite Division of the Institute of Navigation (ION GNSS). Long Beach (CA, USA), 2005, p. 152-155.

[29] SMIDT, J., OZAFRAIN, S., RONCAGLIOLO, P. A., et al. New technique for weak GNSS signal acquisition. IEEE Latin America Transactions, 2014, vol. 12, no. 5. p. 889-894. DOI: 10.1109/TLA.2014.6872901

[30] ZIEDAN, N. I. GNSS Receivers for Weak Signals. Artech House Publishers, 2006. ISBN: 9781596930520

[31] DEDES, G., DEMPSTER, A. G. Indoor GPS positioningchallenges and opportunities. In 2005 IEEE 62nd Vehicular Technology Conference (VTC-2005-Fall). 2005, p. 412-415. DOI: 10.1109/VETECF.2005.1557943

[32] HUAN LI, YUBAI LI, WEI PENG, et al. A novel algorithm for the weak GPS signals acquisition. In The 2nd International Conference on Computer Application and System Modeling. 2012, p. 738-741. DOI: 10.2991/ICCASM.2012.187

[33] PSIAKI, M. L. Block acquisition of weak GPS signals in a software receiver. In Proceedings of ION GPS 2001. Salt Lake City (UT, USA), 2001, p. 2838-2850.

[34] JEON, S., SO, H., KIM, G., et al. Analysis of GNSS signal acquisition methods for the bit-transition problem for a single code period. Transactions of the Japan Society for Aeronautical and Space Sciences, 2013, vol. 56, no. 1, p. 31-41. DOI: $10.2322 /$ tjsass. 56.31

[35] PRESTI, L. L., ZHU, X., FANTINO, M., MULASSANO, P. GNSS signal acquisition in the presence of sign transition. IEEE Journal of Selected Topics in Signal Processing, 2009, vol. 3, no. 4, p. 557-570. DOI: 10.1109/JSTSP.2009.2024592

[36] SUN, K., PRESTI, L. L. Bit sign transition cancellation method for GNSS signal acquisition. The Journal of Navigation, 2012, vol. 65 , no. 1, p. 73-97. DOI: $10.1017 / \mathrm{S} 0373463311000543$

[37] FOUCRAS, M., JULIEN, O., MACABIAU, C., EKAMBI, B. A novel computationally efficient Galileo E1 OS acquisition method for GNSS software receiver. In Proceedings of the 25th International Technical Meeting of the Satellite Division of the Institute of Navigation (ION GNSS 2012). Nashville (TN, USA), 2012, p. 365-383. DOI: $10.13140 /$ RG.2.1.1329.2640

[38] ZHU, C., FAN, X. Weak global navigation satellite system signal acquisition with bit transition. IET Radar Sonar Navigation, 2015, vol. 9, no.1, p. 38-47. DOI: 10.1049/iet-rsn.2013.0253

[39] O'DRISCOLL, C. Performance Analysis of Parallel Acquisition of Weak GPS Signals. Ph.D. Dissertation. National University of Ireland, 2007.

[40] FOUCRAS, M., MACABIAU, C., JULIEN, O., et al. Optimal GNSS acquisition parameters when considering bit transitions. In Position, Location and Navigation Symposium (IEEE/ION PLANS 2014). Monterey (CA, USA), 2014, p. 804-817. DOI: 10.1109/PLANS.2014.6851445

[41] FOUCRAS, M., JULIEN, O., MACABIAU, C., EKAMBI, B., BACARD, F. Probability of detection for GNSS signals with sign transitions. IEEE Transactions on Aerospace and Electronic Systems, 2016, vol. 52, no. 3. p. 1296-1308. DOI: 10.1109/TAES.2016.140316

[42] LANGLEY, R. B. Dilution of precision. GPS World, 1999, vol. 10 , no. 15 , p. $52-59$.
[43] ANGRisano, A., GAGliOne, S., GIOIA, C. Performance assessment of GPS/GLONASS single point positioning in an urban environment. Acta Geodaetica et Geophysica, 2013, vol. 48, no. 2, p. 149-161. DOI: 10.1007/s40328-012-0010-4

[44] CAI, C., GAO, Y. A combined GPS/GLONASS navigation algorithm for use with limited satellite visibility. The Journal of Navigation, 2009, vol. 62, no. 4, p. 671-685. DOI: $10.1017 / \mathrm{S} 0373463309990154$

[45] ANGRisano, A., GAGLIONE, S., GIOIA, C., et al. Testing the test satellites: the GALILEO IOV measurement accuracy. In International Conference on Localization and GNSS (ICL-GNSS). Turin (Italy), 2013, 6 p. DOI: 10.1109/ICL-GNSS.2013.6577253

[46] BetZ, J.W., GOLDSTEIN, D. B. Candidate designs for an additional civil signal in GPS spectral bands. In Proceedings ION NTM 2002, 2002, $10 \mathrm{p}$.

[47] HEIRIES, V., AVILA-RODRIGUEZ, J. A., IRSIGLER, M., et al. Acquisition performance analysis of composite signals for the L1 OS optimized signal. In Proceedings of the 18th International Technical Meeting of the Satellite Division of the Institute of Navigation (ION GNSS 2005).Long Beach (CA, USA), 2005, p. $877-889$.

[48] AVILA-RODRIGUEZ, J. A., HEIN, G. W., WALLNER, S., et al. The MBOC modulation: The final touch to the Galileo frequency and signal plan. Navigation, Journal of the Institute of Navigation, 2007 , vol. 55 , no. 1, p. 15-28. DOI: 10.1002/j.21614296.2008.tb00415.x

[49] MONGREDIEN, C., RÜGAMER, A., OVERBECK, M., et al. Opportunities and challenges for multi-constellation, multifrequency automotive GNSS receivers. In Microelectronic Systems: Circuits, Systems and Applications. 2011, p. 157-172. DOI: 10.1007/978-3-642-23070-7_16

[50] JI, S., CHEN, W., DING, X., CHEN, Y. Potential benefits of GPS/GLONASS/GALILEO integration in an urban canyon Hong Kong. The Journal of Navigation, 2010, vol. 63, no. 4, p. 681-693. DOI: $10.1017 / \mathrm{S} 0373463310000081$

[51] DEVRIES, C. A., MASON, R. D. Subsampling architecture for low power receivers. IEEE Transactions on Circuits and Systems II: Express Briefs, 2008, vol. 55, no. 4, p. 304-308. DOI: 10.1109/TCSII.2008.919495

[52] VAUGHAN, R. G., SCOTT, N. L., WHITE, D. R. The theory of bandpass sampling. IEEE Transactions on Signal Processing, 1991, vol. 39, no. 19, p. 1973-1984. DOI: $10.1109 / 78.134430$

[53] PEKAU, H., HASLETT, J.W. Cascaded noise figure calculations for radio receiver circuits with noise-aliasing properties. IEE Proceedings - Circuits, Devices and Systems, 2006, vol. 153, no. 6, p. 517-524. DOI: 10.1049/ip-cds:20060023

[54] GARCÍA OYA, R. J., KWAN, A., MUNOZ CHAVERO, F. M., GHANNOUCHI, F. M., et al. Subsampling receivers with applications to software defined radio systems. In Data Acquisition Application (INTECH). Ed. Z. Karakehayov. 2012, chapter 7, p. $166-194$. DOI: $10.5772 / 49959$

[55] POULARIKAS, A. D. (Ed.) The Transforms and Applications Handbook. $2^{\text {nd }}$ ed., CRC Press, February 2000, p. 1336. ISBN-10: 0849385954

[56] YAO, Z., LU, M., FUNG, Z. Unambiguous sine-phased binary offset carrier modulated signal acquisition technique. IEEE Transactions on Wireless Communications, 2010. vol. 9, no. 2, p. 577-580. DOI: $10.1109 /$ TWC.2010.02.091066

[57] YAO, Z., CUI, X., LU, M., FENG, Z., YANG, J. Pseudocorrelation function based unambiguous tracking technique for sine-BOC signals. IEEE Transactions on Aerospace and Electronic Systems, 2010, vol. 46, no. 4, p. 1782-1796. DOI: 10.1109/TAES.2010.5595594

[58] IRSIGLER, M., AVILA-RODRIGUEZ, J. A., HEIN, G. W. Criteria for GNSS multipath performance assessment. In 
Proceedings of the 18th International Technical Meeting of the Satellite Division of the Institute of Navigation (ION GNSS 2005). Long Beach (CA, USA), 2005, p. 2166-2177.

\section{About the Authors ...}

Saifeddine CHEBIR was born in Bordj Bou Arreridj (BBA), Algeria on August 30, 1988. He received the License degree in Telecommunications from the University of Bachir El Ibrahimi, BBA, Algeria, in 2009. He obtained his Master degree in Networks and Telecommunications from the same university in 2011. Since 2011, he is with the Dept. of Electronics Engineering of the University of Bachir El Ibrahimi, as a doctorate student in Electrical Engineering and Industrial Data. He works as teacher at the National Inst. of Vocational Training and Education BBA1, BBA, Algeria since 2012. He has joined the Dept. of Electronics at Bachir El Ibrahimi University, in 2013, as a visiting teacher. He is a Member of the ETA Laboratory of the same department since 2015. His research interests include signal processing, communication signals, radionavigation, hybrid satellite communications systems, acquisition and tracking of GNSS signals.

Salih AIDEL was born in Bordj Bou Arréridj, Algeria in 1959. He received the Engineer degree in Electronics from the National Polytechnic School of Algiers (ENPA) in 1985. He obtained his Magister degree in Control and Communication Systems in 1990 from the University of Constantine 1 (UMC), Algeria, and the Ph.D. degree (Doctorat d'Etat) in Electronics Engineering in 2011 from the University of Farhat Abbas, Sétif, Algeria. He joined the Electronics Dept., UFAS1, Sétif, Algeria, in 1985, then he joined the Electronics Dept., University of Bordj Bou Arréridj, Algeria, as an Assistant Professor. Currently, he is the Director of the Electronics and Advanced Communications Laboratory (ETA) since 2015. He conducted many research projects in Machine-to-Machine (M2M) Communications. His research interests include wireless communications, Internet of Things, microwave circuits design, cryptography and network security.

Khaled ROUABAH was born in Bordj Bou Arreridj, Algeria in 1974. He received the Engineer degree in Electronics from the University of Farhat Abbas, Setif, Algeria, in 1999. He obtained his Master degree in Telecommunications and Networking in 2001 from SUPAERO (Ecole Nationale Supérieure de l'Aéronautique et de l'Espace),
Toulouse, France, and his Magister degree in Communications in 2005 from the University of Farhat Abbas, Setif, Algeria, and the Ph.D. degree in Electronics Engineering from the same university in 2010. In 2013, he obtained his HDR degree in Electronics. He joined the Electronics Dept., University of Mohamed Bachir El-Ibrahimi Bordj Bou Arreridj, Algeria, as an Assistant Professor in 2006. Since 2010, he works as a Lecturer at the same department. $\mathrm{He}$ is a Member of the ETA Laboratory of the same department since 2014. He conducted many research projects in Telecommunication by Satellite. His research interests include communication signals, geolocation, parallel processing, hardware implementation, signal structure design, mobile computing and spreading communication.

Salim ATIA was born in Bordj Bou Arreridj Algeria in 1958. He received the Bachelor of Science degree in Electronics in 1981 at the University of Constantine Algeria. In 1984, he received the Master degree in Electrical Engineering from Ohio State University, Ohio USA. He worked as an assistant professor in the Electronics Dept. at the University of Ferhat Abbas Setif Algeria from 1984 to 1991. Then, in 2000 he joined the Electronics Dept. of the University of Mohamed Bachir El-Ibrahimi Bordj Bou Arreridj Algeria, also as an assistant professor. In 2013, he obtained his Ph.D degree in Electronics. He is a member of the ETA laboratory at the University of Mohamed Bachir El-Ibrahimi, Bordj Bou Arreridj, Algeria since 2014. Currently he is preparing a HDR degree in Electronics. His research interests are signal processing of satellite communication, radio communication and radio navigation.

Mustapha FLISSI was born in Setif, Algeria in 1970. He received the B.S. and M.S. degrees in Electronics Engineering from the University of Farhat Abbas, Setif, Algeria, in 1995. He obtained his Magister degree in Communications in 2001 from the University of Farhat Abbas, Setif, Algeria, and the doctorate degree in Electronics from the University of M'sila, Algeria, in 2015. He joined the Electronics Dept., University of Ziyani Achour, Djelfa, Algeria, as an Assistant Professor in 2002. Then, in 2005 he joined the Electronics Dept. of the University of Mohamed Bachir El-Ibrahimi, Bordj Bou Arreridj, Algeria, also as an Assistant Professor. Since 2014, he works as a Lecturer at the same department. He is a Member of ETA Laboratory of the same department since 2015. His research interests include satellite navigation, signal structure design and signal processing. 